\title{
Estimation of manufacturing systems degradation rate for residual life prediction through dynamic workload adjustment
}

\author{
V K MANUPATI ${ }^{1, *}$, SURAJ PANIGRAHI ${ }^{2}$, MUNEEB AHSAN $^{2}$, SOMNATH LAHIRI $^{2}$, \\ AKSHAY CHANDRA ${ }^{2}, \mathrm{~J}_{\mathrm{J}}$ THAKKAR $^{3}$, GORAN PUTNIK ${ }^{4,5}$ and M L R VARELA ${ }^{4,5}$ \\ ${ }^{1}$ Department of Mechanical Engineering, NIT Warangal, Warangal, India \\ ${ }^{2}$ School of Mechanical Engineering, VIT University, Vellore, India \\ ${ }^{3}$ School of Industrial and Systems Engineering, Indian Institute of Technology Kharagpur, Kharagpur, India \\ ${ }^{4}$ Department of Production and Systems, University of Minho, 4800-058 Guimarães, Portugal \\ ${ }^{5}$ Research Centre ALGORITMI, University of Minho, 4800-058 Guimarães, Portugal \\ e-mail: manupati.vijay@nitw.ac.in; suraj.chandrasekhar2015@vit.ac.in; muneeb.ahsan2015@vit.ac.in; \\ somnath.lahiri2015@vit.ac.in; akshay.chandra2015@vit.ac.in; jtiitkgp@gmail.com; putnikgd@dps.uminho.pt; \\ leonilde@dps.uminho.pt
}

MS received 8 January 2018; revised 28 May 2018; accepted 9 July 2018; published online 22 January 2019

\begin{abstract}
Complex systems in a work cell often consist of multiple units to process the manufacturing functions effectively for achieving the desired objectives. All manufacturing work cells are familiar with many unforeseeable events, for instance machine down time and scheduled maintenance. In fact, every configuration naturally exhibits some level of redundancy during those unpredictable events that may fail a small portion of units. In this work, using the remaining units and by raising the workloads on these units, up to the level of their capacities, we tried to fulfil the requirement of products. To procure the requirement, dynamic workload adjustment strategy has been suggested on two important configurations such as parallel and hybrid, by actively controlling its degradation path and failure times. During its operation, at each decision-making point, termed as decision epoch, the examination of the real-time condition monitoring data has been carried out for upgrading the posterior distribution. Using this updated distribution as the root of all operations, the residual life distribution of every concerned unit is calculated, for a particular workload. Subsequently, the establishment of an optimization scheme, i.e., an optimization framework, has been carried out with the help of the predicted residual life to eliminate the unit failures, for individual units, coinciding with each other. Eventually, with various scenarios, simulation has been carried out on the proposed methodology to assess the rate of degradation of various units. The validation of the approach's effectiveness has been shown by the simulation results on two different configurations having different scenarios.
\end{abstract}

Keywords. Residual life prediction; multi-unit systems; simulation; work cell.

\section{Introduction}

The real-life arrangement of complex systems in a work cell usually involves multiple units, each of which functions independently or in union for achieving the desired objectives/outcomes. As a matter of fact, out of various existing configurations, in this paper two important configurations namely parallel and hybrid have been considered as multi-unit systems. In a parallel configuration, multiple units are conditioned to function autonomously and concurrently with each other to satisfy the requirement of the system. For example, an aircraft must be equipped with multiple parallel engines to operate simultaneously for

*For correspondence adequate horsepower generation and for producing sufficient aerodynamic lift. A single-stage manufacturing work cell consists of several identical machines which can perform the same operation not only to fulfil a high production demand but also to attain a smooth flow of production as mentioned in Kaiser and Gebraeel [1]. In the proposed hybrid configuration, the machines are configured as the integration of parallel and series configurations where the multi-unit deemed to function to bring out the efficient and effective results without any mismatch in their operations. For example, Savonius drag-type and Darrieus lift-type rotors have been used as self-sufficient wind turbine-generator systems for efficient electricity production as discussed in Wakui et al [2] and a high-speed optical switch, whose idea has been taken from a generalized Mach- 
Zehnder interferometer, having a hybrid configuration that consists of a silica-based planar lightwave circuit and lithium niobate phase shifters as explained in Suzuki et al [3].

Theoretically, a natural and unavoidable degradation process has to be faced by each concerned unit when performing the operations. As a result, once the predestined threshold of a unit is surpassed by its degradation level, it is reckoned to be failed, for which servicing has to be done on it for it to regain its former state of proper operation. Therefore, when a parallel unit system experiences a unit failure, the remaining efficient units must be allotted with workloads which are comparatively heavier to sustain the system requirements. For example, considering the manufacturing systems, the crest of the production-rate (referring to the capacity) of a machine is designed with heavier than the traditionally allotted workloads, to overcome the undesirable and unexpected or natural events. The U.S. fabricating industries, as reported by the Federal Reserve, are facing nearly $20 \%$ of average redundancy as stated in Otoo and Collins [4]. Practically, when a huge amount of workload is assigned to similar units, they exhibit similar degradation path which leads to overlap of unit failures, ultimately the requirements remaining unsatisfied.

To highlight the issue mentioned above, the main aim of our paper is to avert the cases of unit failure overlap, by developing a dynamic workload adjustment procedure, in both kinds of unit systems - parallel and hybrid. We have taken into consideration the fact that the workload directly influences the degradation rate of a unit; specifically, when a higher workload is applied to an operating unit, then it is considered to degrade at a faster rate. On the basis of this supposition, our fundamental target is to adjust and control the rate of degradation of a unit in real time, by modifying the workload, to predict its failure rate. After all, precise prediction and checking of the failure time of individual units are crucial to forbid the overlap of unit failures, which are significantly beneficial to the manufacturers for maintenance planning, logistics, and to improve productivity.

Extensive use has been made of the degradation modeling and prognostic analysis, in the literature, for a single unit system. However, limited efforts have been made regarding models of statistical and stochastic categories for recognizing the degradation rate of the multi-unit system, which is our present region of focus. Lu and Meeker [5] suggested a degradation model and taking into account nonlinear random-coefficient models, analysis has been performed and then compared with degradation analysis and traditional failure-time analysis regarding asymptotic efficiency. Gebraeel et al [6] suggested a couple of stochastic degradation models which have been used for data obtained from real-time analysis, for updating the model and estimating the RLD (Residual Life Distribution) for units which were partially degraded. Martin [7], Fararooy and Allan [8], Dimla [9] and Thorsen and Dalva [10] provide observation of similar research in some other distinct areas of applications like cutting tools, high-voltage induction motors, railway equipment, machine tools and the like. In their approach, they conducted the literature survey by considering the condition monitoring and degradation modeling, to perceive quantities that can be measured which permit successful diagnosis of the health of a device. As the operating time advances regarding the random coefficients model, various assumptions have been recommended by Wang [11]. Taken into consideration is the device whose degeneration level of its own working condition can be discerned as and when required. Malfunctioning of the device starts as its degradation signal touches a predefined threshold.

To give voice to the challenge, recent research efforts have extended the scope from single unit systems to multiple systems in specific to multiple states as is elaborated in Huang et al [12] and Zuo and Tian [13]. Significant amounts of research works dealt with the relationship between system and unit reliability regarding multi-unit systems. For example, in the $k s$ system, the number of units $n$ must be functional on condition that a minimum of $k$ number of units operate properly. Similar is the case for $k$ out-of- $n$ system as reported in Chao et al [14]. In the same fashion during the inter-dependent degradation processes, for multiple units, the degradation modeling has been considered by Bian and Gebraeel [15]. Unit degradation signals were aimed at for differentiating them from sensor data, constituting various documentation obtained from innumerable related units in a composite system, by Hao et al [16]. These outcomes proved that the contemporary research has primarily focused solely on modeling the degradation and recognizing and preventing the various events of degradation of the units.

Henceforth, in this research, we provide a new comprehension with the aim of actively taking the control of the various steps of degradation of individual units in case of a multi-unit system. To be specific, we recommend to adjust the workload assignment of a unit in parallel and hybrid configurations to control the degradation process. The workloads of individual machines in parallel manufacturing systems are varied, where, if any one of the machines breaks down, its inputs will automatically be redirected and separately assigned to the other functional machines. Sooner or later, these lead to increase in the workload on the remaining existing machines which is elucidated in $\mathrm{Li}$ [17] and Li [18]. Regarding the proposed hybrid manufacturing systems, even if any one of the machines fails to operate, the whole structure will still maintain its stability. The functioning of the units, however, is same as the parallel manufacturing systems. Very few works can be found in the literature associated with the combined effects of degradation and adjustment of workload assignment. An admissible work was cited by Sloan and Shanthikumar [19] and Sloan and Shanthikumar [20], who used a discrete time Markov chain (DTMC) model for unit's degradation and the probabilities for transition from one degradation state to 
the other for dispatching the products to each unit. Similar kind of work is done by Zhou et al [21] for a framework where the operation was transferred among the units to regulate their utilization and thus controlled the respective hazard rates.

For a degenerating manufacturing system related to single machine, both the machine reliability and product quality can be improved by an effective procedure of preventive maintenance (PM) for which quality improvement has been incorporated into PM decision-making in a joint model which has been suggested in Lu et al [22]. A mathematical model associated with both multi-period cell formation and part operation trade-off, included in a dynamic cellular manufacturing system, is suggested in Deep and Singh [23], along with multiple pass process route. Remaining useful life (RUL) has been demonstrated with a two phases data driven procedure in Mosallam et al [24], for both offline and online phase, where it finally utilizes discrete Bayesian filter to eventually approximate the degradation state. Parametric inverse Gaussian (IG) process models are considered for degradation process modeling consisting of constant, monotonic and S-shaped degradation rates, in Peng et al [25], where model parameters for time-varying degradation rates have their physical interpretation highlighted. A review of Condition-based maintenance (CBM) literature has been expressed in Alaswad and Xiang [26], with importance provided to mathematical modelling and optimization approaches. A stochastic and complex environment has been exhibited for taking into account a production planning problem, in Arasteh [27], in which the environment referred to that of electronic equipment production, where, planning produces variable results and also changeable requests. The concept of co-evolution of process planning and configurations of machines, where generation of optimal machine capabilities is carried out through the application of multi-objective genetic algorithms, has been elucidated in Asghar et al [28]. A strategy for resource provisioning and scheduling, designed exclusively for Workflow as a Service (WaaS) environments, has been proposed in Rodriguez and Buyya [29].

A proposal has been put forward by this paper to take into account a stochastic degradation model of the continuous-time continuous-state type to construct a procedure that can potentially control the unit degradation and counteract the superposition of unit failures. This paper extends upon the above mentioned stochastic degradation model proposed by Hao et al [30] who, considering the parallel configuration of units, introduced the instantaneous degradation rate of any individual unit which is characterized by the particular workload applied. However, the recurrent demerits of the above-mentioned journals are: (1) Discrete state models like DTMC or reliability models and such other simplified degradation models are the only models that are considered which were unsuccessful in capturing the continuous degradation process. (2) Only simplified control strategies has been utilized with limited number of control actions. (3) Only parallel configuration was taken into consideration and for all units of the above mentioned configuration taken individually, instantaneous degradation rate was developed which was characterized by the respective workload. In order to solve the above mentioned problem, a hybrid configuration has been developed that ameliorates the dynamic workload adjustment strategy.

In this paper, the concept of degradation modelling was proposed and was extended successfully implemented upon the parallel unit systems by akshay et al [31].The gradual degeneration or breakdown of the units along with the contrast in the degradation processes are encapsulated with the endorsed unit degradation model constituting a linear stochastic differential equation (SDE). For an individual unit, we further presume the degradation rate as a random variable which obeys a distribution pattern, established well in advance, because of material inhomogeneity and manufacturing uncertainty. Here, our main area of focus is the determination of the control variable which is the quantity of workload allotted to each unit per unit time. To accomplish the above objective, with already existing parallel configuration and with proposed hybrid configuration, the analysis has been conducted. First, the analysis of the real-time condition monitoring data has been carried out to update the posterior distribution at each decision epoch. Depending on the distribution which has been upgraded, for a specific workload, the RLD of each unit is calculated. Thereafter, an optimization framework has been further suggested, with the speculated residual life, to inhibit the various cases of unit failure overlap for all the units taken individually. Subsequently, with various scenarios, analysis has been done on the proposed methodology to assess the rate of degradation of various units. The validation of the approach's effectiveness has been shown by the simulation results on two different configurations having different scenarios. The later sections review the detailed description of the proposed framework, method, and analysis.

The remainder of this paper is assembled as follows: Problem formulation is explored in section 2 in which the control variable is to be computed. Section 3 elucidates the dynamic workload adjustment framework. The proposed methodology is explained for unit degradation modeling along which the prediction of residual life is demonstrated in section 4. Regarding a work cell, a real time structure of complex systems been reported using a numerical case study in section 5. Important insights into experiments and procedures are given, and their outcomes are outlined in section 6 . Section 7 provides conclusion and about future works.

\section{Framework of the proposed dynamic workload adjustment}

The proposed methodology that integrates the workload and degradation rates of machines in parallel and hybrid configurations is applied to predict real-time residual life. It 


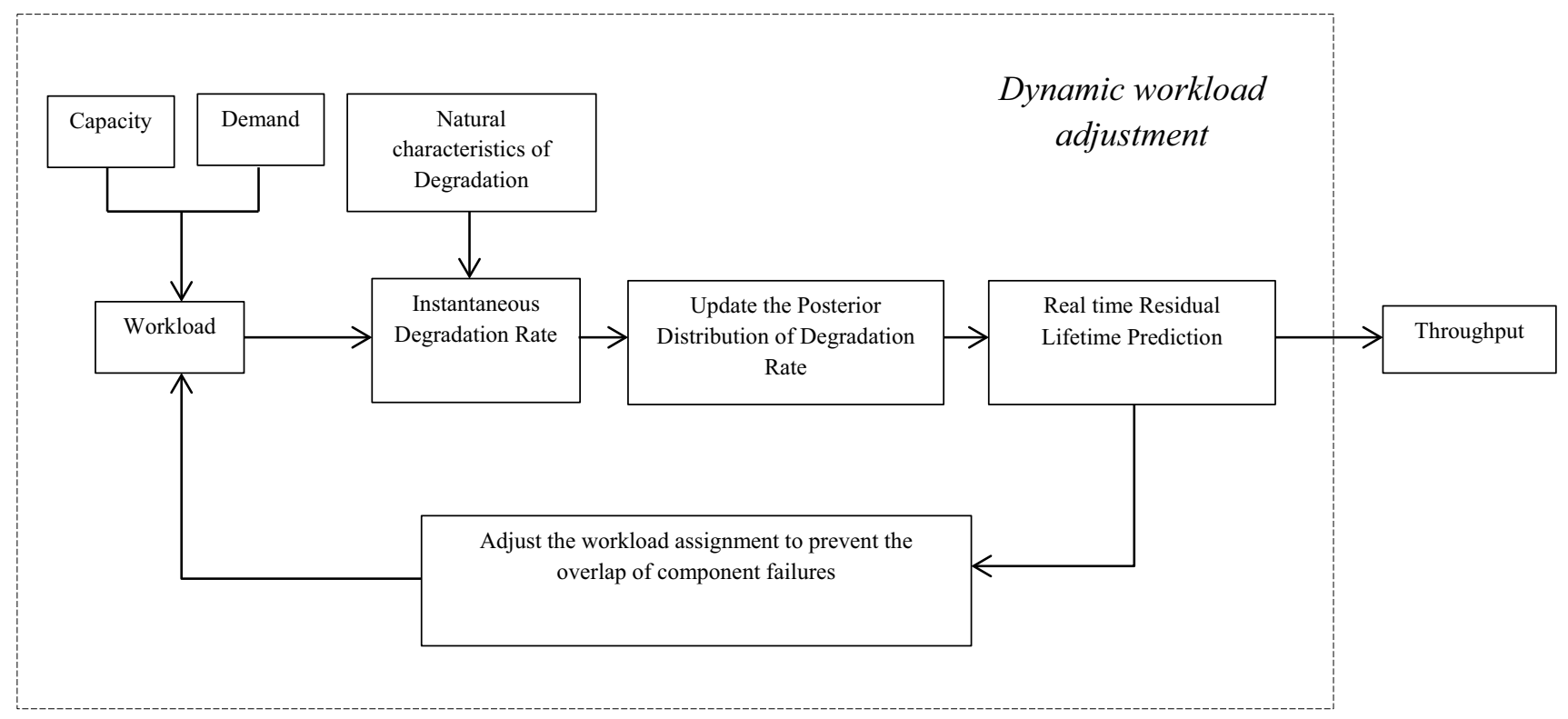

Figure 1. Framework of the proposed methodology.

mainly consists of two parts: the dynamic workload adjustment and the throughput, as illustrated in figure 1. In dynamic workload adjustment, two parameters such as capacity and demand have been considered to assign the respective workloads to individual machines. Here, the total amount of operation at its peak, performed efficiently by each unit in a unit time is termed as its capacity. However, it is noticeable that any machine has its natural degradation rate during its operation which can occur due to several physical and chemical processes such as friction, corrosion, rusting, etc. present in the environment. While conducting the experimentation, the instantaneous degradation rate of each machine and the natural degradation characteristics are taken into account. The posterior distribution is then updated for every trial for each machine to predict realtime residual life. A detailed description of the posterior distribution update of degradation rate is given in section 4.2. After that, the workload adjustment can be done for the overlap of unit failures to be prohibited. Thus, by the desired number of iterations and with the predicted residual life, the net output i.e., "throughput" can be achieved.

For a unit, its residual life can be expressed as the time remaining for its degradation level to reach a pre-defined threshold. For unit $m$, its failure threshold is denoted by $F_{m}$. The residual life of unit $m$ is represented by $T_{m}$. The RLD of unit $m$, which is dependent on the coefficient of degradation $\lambda_{m}$, follows an IG distribution as elaborated in Huang et al [12] as

$$
P\left(T_{m} \leq t \mid A_{m}\left(t_{x}\right), w_{m}\left(t_{x}\right), \lambda_{m}\right) \sim I G\left(t ; \mu_{m}\left(t_{x}\right), S_{m}\left(t_{x}\right)\right)
$$

where $\operatorname{IG}(t ; . .$.$) denotes the cumulative distribution function$ of an IG distribution, $\mu_{m}\left(t_{x}\right)=\left(F_{m}-A_{m}\left(t_{x}\right)\right) /\left(\lambda_{m} w_{m}\left(t_{x}\right)\right)$ is considered to be the mean parameter of an IG distribution whereas $S_{m}\left(t_{x}\right)=\left(\left[F_{m}-A_{m}\left(t_{x}\right)\right]^{2}\right) /\left(D_{m}^{2}\right)$ is the shape parameter of an IG distribution. In this case, note that $w_{m}\left(t_{x}\right)$ is the lately assigned workload. Even though the conditional RLD is associated with Eq. (1), but the unconditional RLD has no direct expression which can be numerically calculated using a simulation approach as discussed in Bian and Gebraeel [15]. This approach can be used to calculate the total number of products and their make span at the end of the simulation period. The conditional RLD is the main area of focus and we make use of $\beta_{m}\left(t_{x}\right)$ as the point estimator of $\lambda_{m}$. Thus, the mean parameter of an IG distribution, $\mu_{m}\left(t_{x}\right)$ in Eq. (1), is approximated by $\mu_{m}\left(t_{x}\right)=\left(F_{m}-A_{m}\left(t_{x}\right)\right) /\left(\beta_{m}\left(t_{x}\right) w_{m}\left(t_{x}\right)\right)$. Here, $\mu_{m}\left(t_{x}\right)$ represents an approximation of the predicted residual life. Elwany and Gebraeel [32] also dealt with this proposal which proved efficient in demonstrating the technique by which attaining a conservative lower bound of the mean of the unconditional RLD can be made possible. For the ease in dealing with notations, we express $d i_{m}\left(t_{x}\right)=$ $\left(F_{m}-A_{m}\left(t_{x}\right)\right) /\left(\beta_{m}\left(t_{x}\right)\right)$ as the health status of the operational unit $m$ at time $t_{x}$. Therefore, we get

$$
\mu_{m}\left(t_{x}\right)=\frac{d i_{m}\left(t_{x}\right)}{w_{m}\left(t_{x}\right)}
$$

From Eq. (2), it is observed that keeping the workload assignment $w_{m}\left(t_{x}\right)$ constant, a smaller value of $d i_{m}\left(t_{x}\right)$ signifies a worse health status and a reduced residual life. Regarding the limiting case, $d i_{m}\left(t_{x}\right)=0$ indicates the occurrence of a failure. Also, in case of $d i_{m}\left(t_{x}\right)$ having some nonzero value, $\mu_{m}\left(t_{x}\right)$, referred to as the predicted residual life, varies inversely as the actual workload. Depending on 


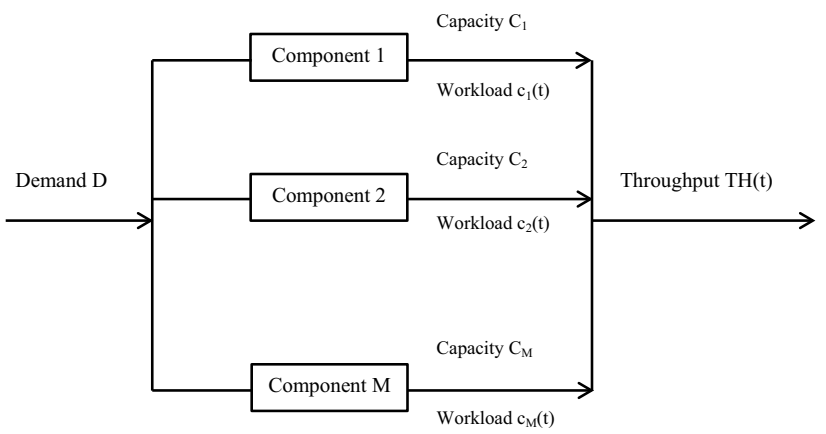

Figure 2. Parallel configuration.

such outcomes, the dynamic control of the residual life by workload adjustment is provided with a heuristic strategy.

\section{Problem description}

We consider parallel and hybrid configurations as unit systems, as illustrated in figures 2 and 3 that consist of $M$ units to perform operations which are identical in nature. Each operation can be assigned to any unit to be performed. The total amount of operations allotted to each unit in a unit time is the control variable whose determination is the foremost domain in this paper. "Capacity" of a particular unit is defined as the maximum amount of operations that are performed by it per unit time, here it is represented by $C_{m}$ for unit $m$. The actual amount of operations that each unit performs in a unit time is termed as the "workload", which is represented by $w_{m}(t)$ for unit $m$ at time $t$. This indicates that the workload $w_{m}(t)$ being the control variable that is to be discerned in this paper. By default, the span of workload is set as $0 \leq w_{m}(t) \leq C_{m}$, for $m=1,2, \ldots, M$. If, by any reason, failure is experienced by unit $m$ at time $t$, then $w_{m}(t)=0$. Considering the system, its "throughput rate" at time $t$ is symbolized by $\mathrm{TH}(t)$ and is defined as the total workload from all the units, i.e., $\mathrm{TH}(t)=\sum_{m=1}^{\tilde{n}_{u}(t)} C_{m}(t)$. Let $\tilde{n}_{u}(t)$ be the number of units which are functional at time $t$. Then "capacity", referred to as the peak throughput rate, of the system at time $t$ is equal to $\sum_{m=1}^{\tilde{n}_{u}(t)} C_{m}$. Note that $T H(t)=\min \left[\sum_{m=1}^{\tilde{n}_{u}(t)} C_{m}, D\right]$, where, the "demand" is specified by $D$. When $\sum_{m=1}^{\tilde{n}_{u}(t)} C_{m} \geq D$, then $T H(t)=D$. This implies that when the capacity of the system surpasses the demand, the throughput rate of the system equals to the demand. However, when $\sum_{m=1}^{\tilde{n}_{u}(t)} C_{m} \leq D$, then $T H(t)=\sum_{m=1}^{\tilde{n}_{u}(t)} C_{m}$. This states that, regarding the case of the remaining functional units, when their capacity is lower than the demand, the system produces the output as the maximum possible throughput rate which equals to the capacity of the operational units. To make this point clear, the demand is assumed to be constant and the operations performed by the units are deemed to be identical in nature. It is important to note that workload, throughput rate, demand and capacity have their measurement units which may either be continuous or discrete, according to their dependence upon the different application areas.

Assumptions:

(1) We have considered linear degradation model which is elucidated by Gebraeel et al [6] where degradation rate of a unit is directly proportional to its workload. It means a unit functioning under a higher workload is assumed to degrade faster and vice versa.

$$
d A_{m}(t)=\lambda_{m} w_{m}(t) d t+d W_{m}(t)
$$

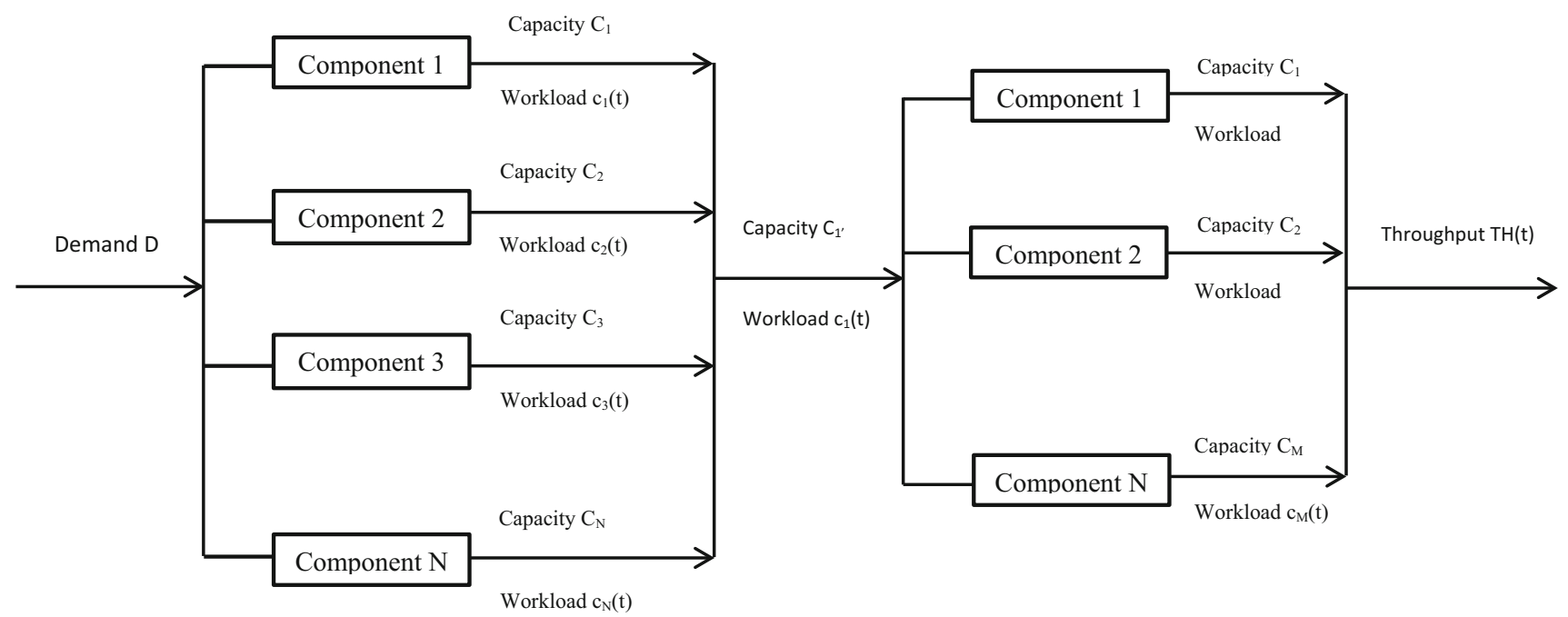

Figure 3. Hybrid. 
(2) Only one type of operation is performed by the units, and the demand is considered to be constant.

(3) The actual value of $\lambda_{m}$ is unknown and random. To capture the variation in the degradation processes due to material inhomogeneity and other manufacturing related uncertainty we assume "unit to unit variability" to be widely adopted in this paper.

(4) Unit failure due to unsatisfactory quality of the performed operations is not considered in this paper, only due to its own degradation rate is considered.

(5) The operation of a product on a machine should not be obstructed until it is completed.

\subsection{Mathematical modeling}

Minimization of Throughput time

$$
\mathrm{Z}=\frac{1}{\widetilde{n_{u}}\left(t_{x}\right)} \sum_{m=1}^{n_{u}\left(t_{x}\right)}\left[\beta_{(m)}\left(t_{x}\right) w_{(m)}\left(t_{x}\right) \delta t+A_{(m)}\left(t_{x}\right)\right]
$$

subject to the following constraints:

$$
\begin{gathered}
\sum_{m=1}^{\tilde{n}\left(t_{x}\right)} w_{(m)}\left(t_{x}\right)=\min \left(\sum_{m=1}^{\tilde{n}\left(t_{x}\right)} w_{m}, D\right) \\
w_{(1)} t_{(x)} \geq \ldots \geq w_{\left(\tilde{n}_{u}\left(t_{x}\right)\right)}\left(t_{x}\right) \\
0 \leq w_{m}\left(t_{x}\right) \leq C_{m}, \quad \text { for } \mathrm{m}=1, \ldots, \mathrm{M} \\
\frac{d_{i(m)}\left(t_{x}\right)}{w_{m}\left(t_{x}\right)}+R_{(m)} \delta t \leq \frac{d_{i(m+1)} t_{(x)}}{w_{(m+1)}\left(t_{x}\right)} \quad \text { for } \mathrm{m}=1, \ldots, \tilde{n}_{u}\left(t_{x}\right)-1
\end{gathered}
$$

Equation (3) through its objective function, assures that overall, failure of all units should happen in the slowest pace as for the system, it is an indication of its throughput time. In real time, the objective value on the health status of the system provides an insight into the above stated procedure. Constraint (4) assures that, throughput of the concerned system equals its demand when the demand is less then its capacity. On the other hand, its throughput is set according to its capacity, when its demand has a higher value than its capacity. Constraint (5) addresses the allotting of higher workloads to the relatively more degraded units, i.e., the units having degradation status which are more acute. Constraint (6) describes the circumstance, where the workload allotted to the respective machines is a nonnegative quantity and always has a lesser value than the peak capacity of the machines. Constraint (7) details the prevention of the cases of unit failure overlap, which means that the difference between the speculated residual lives of any two units which will face failure successively, must have a higher value than the repair time of the units separately.

\section{Unit degradation modeling}

\subsection{Degradation modeling framework}

Machines degrade while performing their specified operations, which is natural as degradation is an inevitable procedure that takes place progressively over a span of time. A unit is deemed to have failed, when a pre-defined failure threshold of the concerned unit is surpassed by the degradation level of the unit. In this paper, to determine the predefined failure threshold, exploitation of either industrial standards, as detailed in Gebraeel et al [33], or ideas based on data-driven approaches, as expressed in Liu et al [34], has been done. Once a unit is supposed to be failed, it should be sent for repairing or replacement before restarting the next operation, for it to be restored to its original performance mode.

Catastrophic failure, cascading systems failure and likewise countless other types of distinct failures prevail in reality. In this paper, the sole unit failure taken into consideration is the failure due to degradation. Nevertheless, we intend to have the above-mentioned failures and ways to overcome them, in our future works. Degradation of a unit could lead to the quality of the performed operations being easily affected. Anyway, the reason considered for unit failure is self-degradation of the units and not inefficient quality of the performed operations. A necessary factor for developing the link between unit degradation and quality of operations is the in-depth knowledge which is required on mathematical modeling and system configurations. The amplitude of the degradation signal of unit $m$ at time $t$ is denoted by $A_{m}(t)$. Considering $A_{m}(t)$ as an SDE, a generic degradation model is developed as

$$
d A_{m}(t)=i_{m}(t) d t+d W_{m}(t)
$$

where $i_{m}(t)$ represents instantaneous degradation rate, and $W_{m}(t)$ represents the Brownian motion with variance $d_{m}^{2} t$, where $d_{m}$ denotes the diffusion parameter. Continuous stochastic degradation model of such type has been substantially used to mathematically explain the procedure of degradation of any unit.

In the suggested work, the association that the instantaneous degradation rate $i_{m}(t)$ has with the workload $w_{m}(t)$, has been explicitly modeled for unit $m$ at time $t$. Theoretically, this task is remarkably challenging because of its high dependence on the definite applications and various working conditions. Previously, efforts have been made in many research areas, to specify the relationship that the working condition has with degradation rate, through historical data by making use of authentic mathematical assumptions. For example, Bian and Gebraeel [35] 


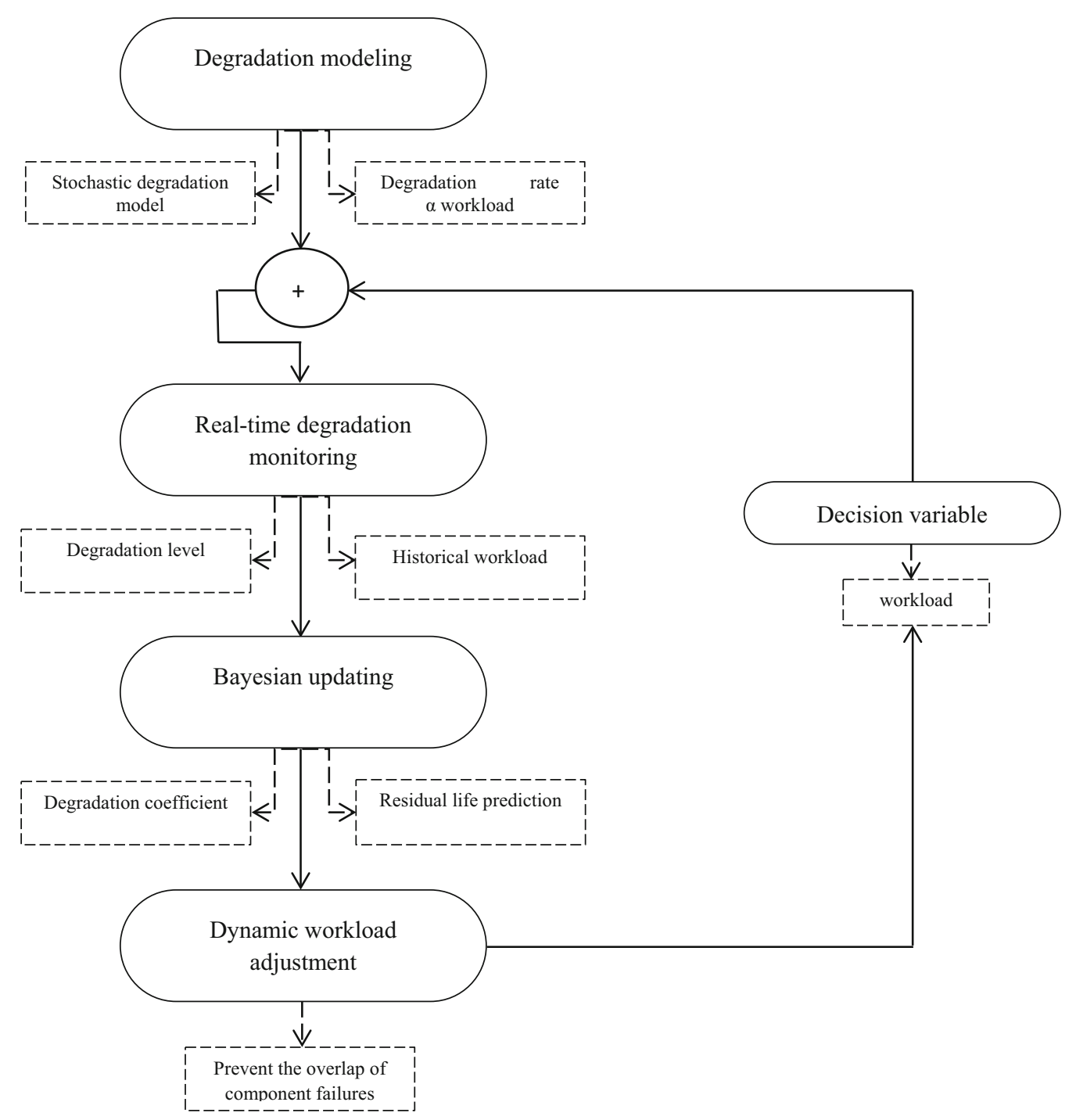

Figure 4. Extended approach of the proposed methodology.

established an experiment to monitor the degradation signals of ball bearings subjected to distinct values of load and speed. The results of their experiment implied that as the speed or load increases, the degradation rate will definitely increase and the lifetime will certainly decrease. The novelty of our work is to gather knowledge on developing a workload adjustment strategy, as shown in figure 4 , to restrict the stages of degradation of the units for which this relationship function is defined. Here, we concentrate on the factor of the instantaneous degradation rate $i_{m}(t)$ being directly proportional to the applied workload $w_{m}(t)$, as

$$
i_{m}(t)=\lambda_{m} w_{m}(t)
$$

where $\lambda_{m}$ is attributed as the degradation coefficient of unit $m$. This linear assumption is precisely matched with our instinct since the degradation of a unit is faster as higher workload is applied on it. Substituting Eq. (9) into Eq. (8), the degradation model in Eq. (8) can be modified as

$$
d A_{m}(t)=\lambda_{m} w_{m}(t) d t+d W_{m}(t)
$$

In this paper, the exact value of $\lambda_{m}$ is assumed to be indefinite and arbitrary. The supposition of "unit to unit variability" has been used on a large scale to demonstrate the variation in degradation processes because of material in-homogeneity and other uncertainties related to manufacturing aspects. Particularly, $\lambda_{m}$ has been characterized as a random variable, with its prior distribution being set as a normal distribution: $\lambda_{m} \sim N\left(m, \gamma_{m}^{2}\right)$, where $m$ and $\gamma_{m}^{2}$ represent the mean and variance, respectively. In this case, the probability of $\lambda_{m} \leq 0$ is trivial and therefore is ignored in this paper. 


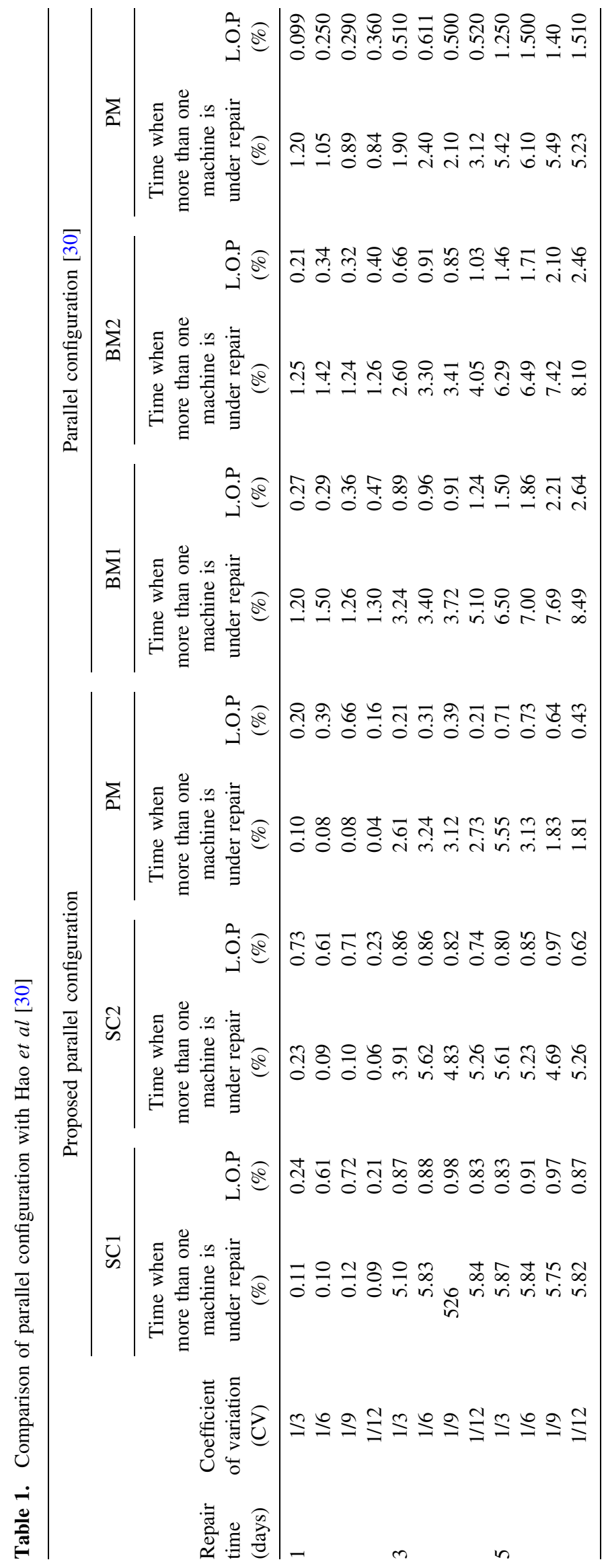




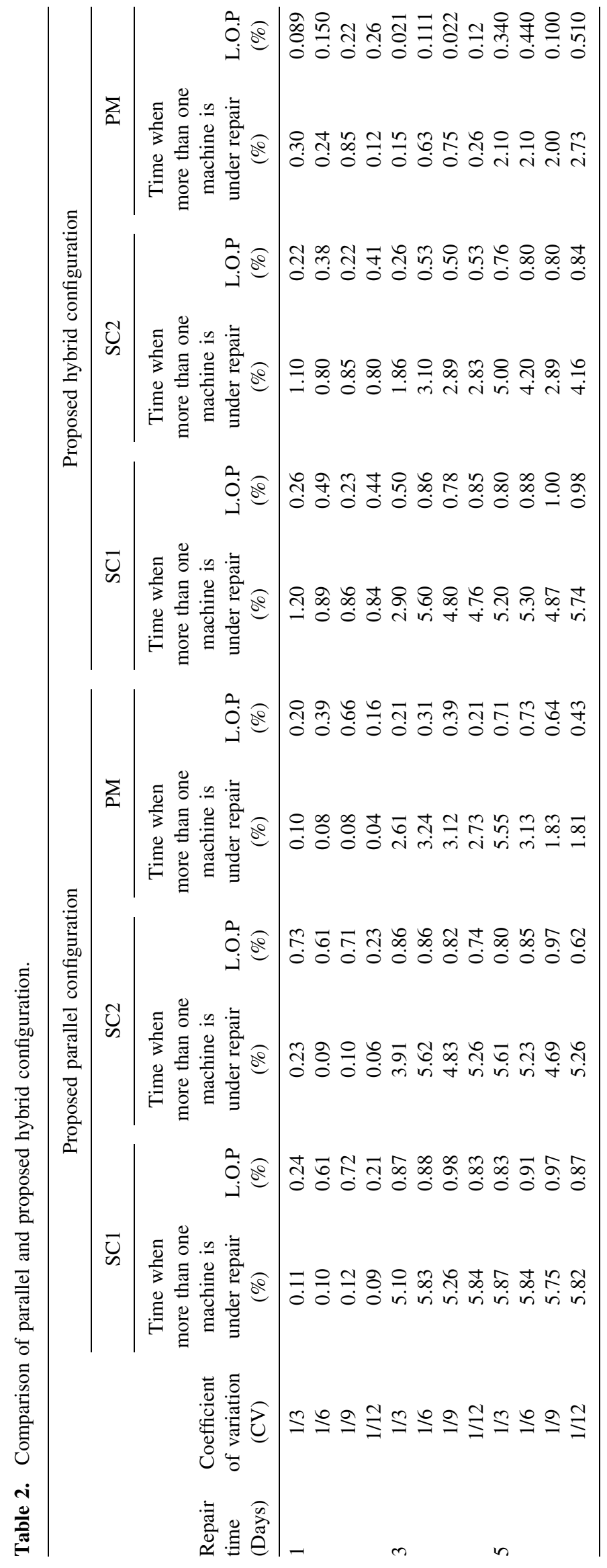




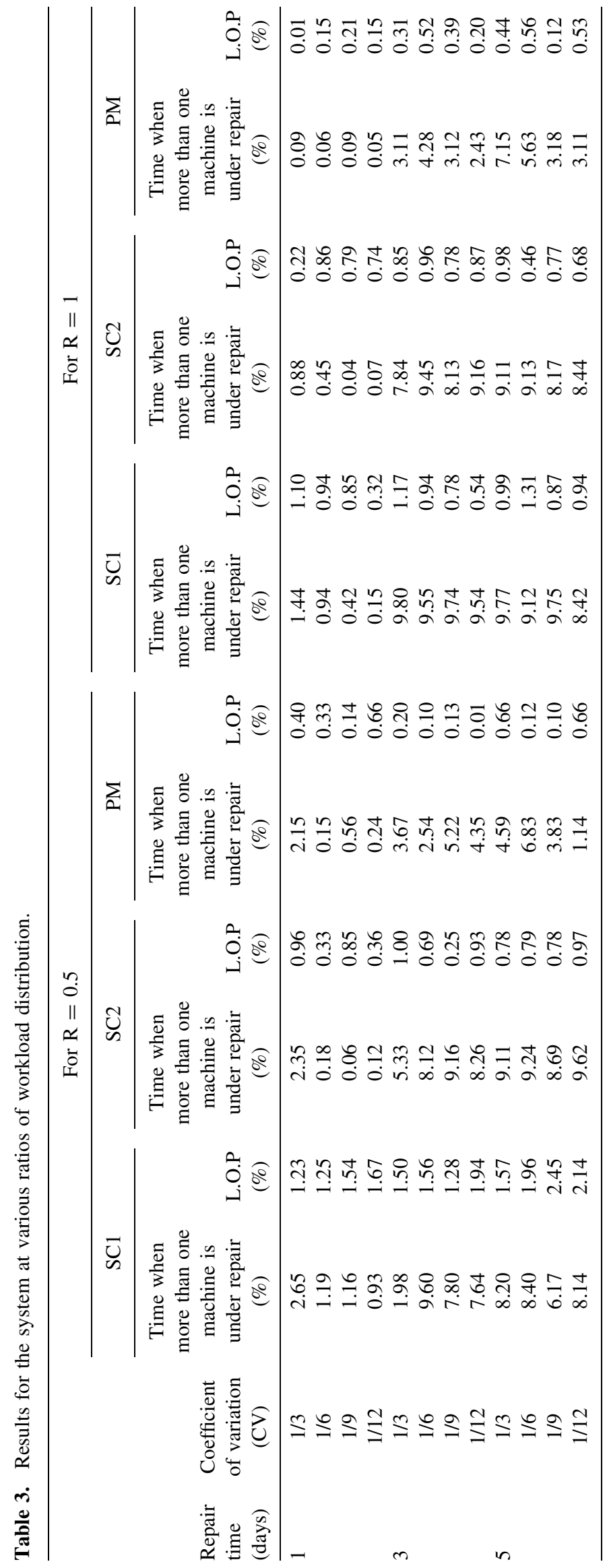




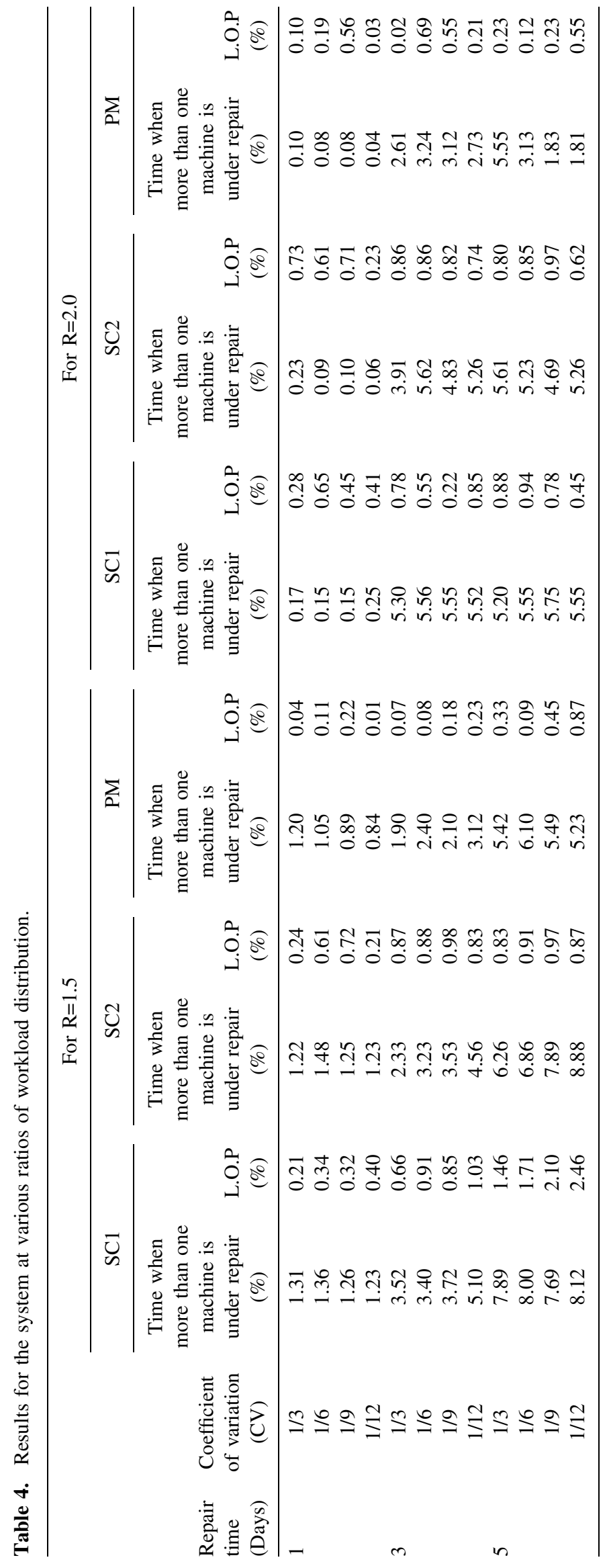


Table 5. The notations used to explain the proposed model.

\begin{tabular}{|c|c|}
\hline$\overline{n_{u}}$ & Number of units \\
\hline$D$ & Demand per unit time \\
\hline$C_{m}$ & Capacity of unit $m$ \\
\hline$w_{m}(t)$ & Workload of unit $m$ at time $t$ \\
\hline$T H(t)$ & Throughput rate of the system at time $t$ \\
\hline$\widetilde{n_{u}}(t)$ & Number of functional units at time $t$ \\
\hline$A_{m}(t)$ & Amplitude of the degradation signal of unit $m$ at time $t$ \\
\hline$i_{m}(t)$ & Instantaneous degradation rate of unit $m$ at time $t$ \\
\hline$W_{m}(t)$ & Brownian motion degradation error of unit $m$ at time $t$ \\
\hline$D_{m}$ & Diffusion parameter of $W_{m}(t)$ \\
\hline$\lambda_{m}$ & Degradation coefficient for unit $m$ \\
\hline$\beta_{m}$ & Mean of the prior distribution of $\lambda_{m}$ \\
\hline$\gamma_{m}^{2}$ & Variance of the prior distribution of $\lambda_{m}$ \\
\hline$\delta t$ & Sampling interval \\
\hline$W_{m}\left(t_{x-1}\right)$ & $\begin{array}{l}\text { Column vector that represents the workloads of unit } \\
\qquad m \text { from } t_{0} \text { to } t_{x-1} .\end{array}$ \\
\hline$\delta A_{m}\left(t_{x}\right)$ & $\begin{array}{l}\text { Column vector that represents degradation increments } \\
\text { of unit } m \text { observed from to } t_{x-1}\end{array}$ \\
\hline$p()$. & Probability density function of a distribution \\
\hline$\beta_{m}\left(t_{x}\right)$ & Mean of the posterior distribution of $\lambda_{m}$ updated at $t_{x}$ \\
\hline$\gamma_{m}^{2}\left(t_{x}\right)$ & $\begin{array}{l}\text { Variance of the posterior distribution of } \lambda_{m} \text { updated at } \\
\qquad t_{x}\end{array}$ \\
\hline$F_{m}$ & Pre-defined failure threshold for unit $m$ \\
\hline$I G()$. & $\begin{array}{l}\text { Cumulative distribution function of an inverse } \\
\text { Gaussian distribution }\end{array}$ \\
\hline$\mu_{m}\left(t_{x}\right)$ & $\begin{array}{l}\text { Mean parameter of the conditional residual life } \\
\text { distribution of unit } m \text { computed at } t_{x}\end{array}$ \\
\hline$S_{m}\left(t_{x}\right)$ & $\begin{array}{l}\text { Shape parameter of the conditional residual life } \\
\text { distribution of unit } m \text { computed at } t_{x}\end{array}$ \\
\hline$d i_{m}\left(t_{x}\right)$ & $\begin{array}{c}\text { "Degradation indicator" of unit } m \text { which is related to } \\
\text { the severity of degradation }\end{array}$ \\
\hline$R_{m} \delta t$ & Repair time of unit $m$ \\
\hline
\end{tabular}

The following section will illustrate how the upgradation of the prior distribution of $\lambda_{m}$ has been carried out by making use of the real-time measurements assembled from condition monitoring through a Bayesian approach.

\subsection{Unit degradation model update}

As stated above, in situ monitoring data is used to update the degradation model in Eq. (10). Our main objective is to construct the latest unit degradation status to refine the technique of framing decisions concerning the adjustment procedures of workload. The performance of workload adjustment and condition monitoring at discrete observation epochs $t_{0}, t_{1}, \ldots, t_{x}$, is assumed in this paper. Here, $t_{x}$ denotes the latest observation epoch. The sampling intervals are kept constant, i.e., $t_{1}-t_{0}=t_{2}-t_{1}=\ldots$ $=t_{x}-t_{x-1}=\delta t$. Further, $A_{m}\left(t_{x}\right)$ is taken as the amplitude of the degradation signal of unit $m$ at observation time $t_{x}$, and parallelly the workload that is allotted to the mentioned unit during the interval $\left(t_{x-1}, t_{x}\right)$ is $\delta A_{m}\left(t_{x}\right)=A_{m}\left(t_{x}\right)-$
$A_{m}\left(t_{x-1}\right)$. According to the characteristics of Brownian motion, we have $W_{m}\left(t_{k}\right)-W_{m}\left(t_{k-1}\right) \sim N\left(0, D_{m}^{2} \delta t\right)$. Therefore, once the respective workload assignment $w_{m}\left(t_{x-1}\right)$ and degradation coefficient $\lambda_{m}$ are given, the conditional distribution of $\delta A_{m}\left(t_{x}\right)$ continues to follow a normal distribution $\delta A_{m}\left(t_{x}\right) \mid w_{m}\left(t_{x-1}\right), \lambda_{m} \sim N\left(\lambda_{m} w_{m}\left(t_{x-1}\right) \delta t, D_{m}^{2} \delta t\right)$. The property of unconventional increment of the Brownian motion states that $\delta A_{m}\left(t_{1}\right), \ldots, \delta A_{m}\left(t_{x}\right)$ are statistically independent. Hence, the probability density function of the increments of the signal is represented as follows: where $\delta A_{m}\left(t_{x}\right)=\left[\delta A_{m}\left(t_{1}\right), \ldots ., \delta A_{m}\left(t_{x}\right)\right]$ and $w_{m}\left(t_{x-1}\right)=\left[w_{m}\right.$ $\left.\left(t_{0}\right), \ldots, w_{m}\left(t_{x-1}\right)\right]$. Hence the computation of $\delta A_{m}\left(t_{x}\right)$ and $w_{m}\left(t_{x-1}\right)$ can be done with the help of Proposition 1. Then, the posterior distribution of $\lambda_{m}$ can is obtained.

Proposition $1 \quad \lambda_{m} \mid \delta A_{m}\left(t_{x}\right), w_{m}\left(t_{x-1}\right)$ follows a normal distribution with mean $\beta_{m}\left(t_{x}\right)$ and variance $\gamma_{m}^{2}\left(t_{x}\right)$ as

$$
\begin{aligned}
\beta_{m}\left(t_{x}\right) & =\frac{\left(\gamma_{m}^{2} \sum_{i=1}^{x} \delta A_{m}\left(t_{i}\right) w_{m}\left(t_{i-1}\right)+\beta_{m} d_{m}^{2}\right)}{\left(\gamma_{m}^{2} \sum_{i=1}^{x}\left[w_{m}\left(t_{i-1}\right)\right]^{2} \delta t+d_{m}^{2}\right)} \\
\gamma_{m}^{2}\left(t_{x}\right) & =\frac{d_{m}^{2} \gamma_{m}^{2}}{\gamma_{m}^{2} \sum_{i=1}^{x}\left[w_{m}\left(t_{i-1}\right)\right]^{2} \delta t+d_{m}^{2}}
\end{aligned}
$$

Here, the probability of $\lambda_{m} \mid \delta A_{m}\left(t_{x}\right), w_{m}\left(t_{x-1}\right) \leq 0$ is usually very small and thus is not considered in the paper.

Proof The function $p\left(\lambda_{m} \mid \delta A_{m}\left(t_{x}\right), w_{m}\left(t_{x-1}\right)\right) \alpha p\left(\delta A_{m}\left(t_{x}\right) \mid\right.$ $\left.w_{m}\left(t_{x-1}\right), \lambda_{m}\right) p\left(\lambda_{m}\right)$ is based on the concept of conditional probability. Where $\prod_{i=1}^{x} p\left(\delta A_{m}\left(t_{i}\right) \mid w_{m}\left(t_{i-1}\right), \lambda_{m}\right) p\left(\lambda_{m}\right)$ is related to the idea of probability density function. The concept of normal distribution was used to get the proof of this proposition.

$$
\begin{aligned}
& p\left(\lambda_{m} \mid \delta A_{m}\left(t_{x}\right), w_{m}\left(t_{x-1}\right)\right) \\
& \alpha p\left(\delta A_{m}\left(t_{x}\right) \mid w_{m}\left(t_{x-1}\right), \lambda_{m}\right) p\left(\lambda_{m}\right) \\
& \alpha \prod_{i=1}^{x} p\left(\delta A_{m}\left(t_{i}\right) \mid w_{m}\left(t_{i-1}\right), \lambda_{m}\right) p\left(\lambda_{m}\right) \\
& \alpha \exp \left\{-\sum_{i=1}^{x} \frac{\left[\delta A_{m}\left(t_{i}\right)-\lambda_{m} w_{m}\left(t_{i-1}\right) \delta t\right]^{2}}{2 d_{m}^{2} \delta t}-\frac{\left[\lambda_{m}-\beta_{m}\right]^{2}}{2 \gamma_{m}^{2}}\right\} \\
& \alpha \exp \left\{\frac{-1}{2}\left[\frac{\sum_{i=1}^{x}\left[w_{m}\left(t_{i-1}\right)\right]^{2} \delta t}{d_{m}^{2}}+\frac{1}{\gamma_{m}^{2}}\right] \lambda_{m}^{2}\right\} \\
& \times \exp \left\{\left[\frac{\sum_{i=1}^{x} \delta A_{m}\left(t_{i}\right) w_{m}\left(t_{i-1}\right)}{d_{m}^{2}}+\frac{\beta_{m}}{\gamma_{m}^{2}}\right] \lambda_{m}^{2}\right\}
\end{aligned}
$$




$$
\alpha \exp \left\{-\frac{\left[\lambda_{m}-\frac{\gamma_{m}^{2} \sum_{i=1}^{x} \delta A_{m}\left(t_{i}\right) w_{m}\left(t_{i-1}\right)+\beta_{m} d_{m}^{2}}{\gamma_{m}^{2} \sum_{i=1}^{x}\left[w_{m}\left(t_{i-1}\right)\right]^{2} \delta t+d_{m}^{2}}\right]^{2}}{2 d_{m}^{2} \gamma_{m}^{2}}\right\}
$$

for $\mathrm{t}_{x}$

$$
\begin{aligned}
& \alpha N\left(\beta_{m}\left(t_{x}\right), \gamma_{m}^{2}\left(t_{x}\right)\right) \\
& \beta_{m}\left(t_{x}\right)=\frac{\left(\gamma_{m}^{2} \sum_{i=1}^{x} \delta A_{m}\left(t_{i}\right) w_{m}\left(t_{i-1}\right)+\beta_{m} d_{m}^{2}\right)}{\left(\gamma_{m}^{2} \sum_{i=1}^{x}\left[w_{m}\left(t_{i-1}\right)\right]^{2} \delta t+d_{m}^{2}\right)} \\
& \gamma_{m}^{2}\left(t_{x}\right)=\frac{d_{m}^{2} \gamma_{m}^{2}}{\gamma_{m}^{2} \sum_{i=1}^{x}\left[w_{m}\left(t_{i-1}\right)\right]^{2} \delta t+d_{m}^{2}}
\end{aligned}
$$

Here, $\lambda_{m} \mid \delta A_{m}\left(t_{x}\right), w_{m}\left(t_{x-1}\right), \beta_{m}\left(t_{x}\right), \gamma_{m}^{2}\left(t_{x}\right)$ are as expressed in the proposition. Note that the last equation is the normal density function. Thus $\left(\lambda_{m} \mid \delta A_{m}\left(t_{x}\right), w_{m}\left(t_{x-1}\right)\right)$ has a normal posterior distribution with $\left(\beta_{m}\left(t_{x}\right), \gamma_{m}^{2}\left(t_{x}\right)\right)$ as mean and variance, respectively.

Proposition $2 \lambda_{m, n} \mid \delta A_{m, n}\left(t_{x}\right), w_{m, n}\left(t_{x-1}\right)$ follows a normal distribution with mean $\beta_{m, n}\left(t_{x}\right)$ moreover, variance $\gamma_{m, n}^{2}\left(t_{x}\right)$ as

$$
\begin{gathered}
\beta_{m}\left(t_{x}\right)=\frac{\left(\gamma_{m}^{2} \sum_{i=1}^{x} \delta A_{m}\left(t_{i}\right) w_{m}\left(t_{i-1}\right)+\beta_{m} d_{m}^{2}\right)}{\left(\gamma_{m}^{2} \sum_{i=1}^{x}\left[w_{m}\left(t_{i-1}\right)\right]^{2} \delta t+d_{m}^{2}\right)} \\
\beta_{n}\left(t_{x}\right)=\frac{\left(\gamma_{n}^{2} \sum_{i=1}^{x} \delta A_{n}\left(t_{i}\right) w_{n}\left(t_{i-1}\right)+\beta_{n} d_{n}^{2}\right)}{\left(\gamma_{n}^{2} \sum_{i=1}^{x}\left[w_{n}\left(t_{i-1}\right)\right]^{2} \delta t+d_{n}^{2}\right)} \\
\gamma_{m}^{2}\left(t_{x}\right)=\frac{d_{m}^{2} \gamma_{m}^{2}}{\gamma_{m}^{2} \sum_{i=1}^{x}\left[w_{m}\left(t_{i-1}\right)\right]^{2} \delta t+d_{m}^{2}} \\
\gamma_{n}^{2}\left(t_{x}\right)=\frac{d_{n}^{2} \gamma_{n}^{2}}{\gamma_{n}^{2} \sum_{i=1}^{x}\left[w_{n}\left(t_{i-1}\right)\right]^{2} \delta t+d_{n}^{2}}
\end{gathered}
$$

Here, the probability of $\lambda_{m, n} \mid \delta A_{m, n}\left(t_{x}\right), w_{m, n}\left(t_{x-1}\right) \leq 0$ is usually very small and is not considered in the paper.

Proof The function $p\left(\lambda_{m, n} \mid \delta A_{m, n}\left(t_{x}\right), w_{m, n}\left(t_{x-1}\right)\right) \alpha p\left(\delta A_{m, n}\right.$ $\left.\left(t_{x}\right) \mid w_{m, n}\left(t_{x-1}\right), \lambda_{m, n}\right) p\left(\lambda_{m, n}\right)$ is based on the concept of conditional probability. Where $\prod_{i=1}^{x} p\left(\delta A_{m, n}\left(t_{i}\right) \mid w_{m, n}\right.$ $\left.\left(t_{i-1}\right), \lambda_{m, n}\right) p\left(\lambda_{m, n}\right)$ is related to the idea of probability density function. The concept of normal distribution was used to get the proof of this proposition.

$$
\begin{aligned}
& p\left(\lambda_{m, n} \mid \delta A_{m, n}\left(t_{x}\right), w_{m, n}\left(t_{x-1}\right)\right) \\
& \alpha p\left(\delta A_{m, n}\left(t_{x}\right) \mid w_{m, n}\left(t_{x-1}\right), \lambda_{m, n}\right) p\left(\lambda_{m, n}\right) \\
& \alpha \prod_{i=1}^{x} p\left(\delta A_{m}\left(t_{i}\right) \mid w_{m}\left(t_{i-1}\right), \lambda_{m}\right) p\left(\lambda_{m}\right) \\
& \times \prod_{i=1}^{x} p\left(\delta A_{n}\left(t_{i}\right) \mid w_{n}\left(t_{i-1}\right), \lambda_{n}\right) p\left(\lambda_{n}\right) \\
& \alpha \exp \left\{-\sum_{i=1}^{x} \frac{\left[\delta A_{m}\left(t_{i}\right)-\lambda_{m} w_{m}\left(t_{i-1}\right) \delta t\right]^{2}}{2 d_{m}^{2} \delta t}-\frac{\left[\lambda_{m}-\beta_{m}\right]^{2}}{2 \gamma_{m}^{2}}\right\} \\
& \quad \times \exp \left\{-\sum_{i=1}^{x} \frac{\left[\delta A_{n}\left(t_{i}\right)-\lambda_{n} w_{n}\left(t_{i-1}\right) \delta t\right]^{2}}{2 d_{n}^{2} \delta t}-\frac{\left[\lambda_{n}-\beta_{n}\right]^{2}}{2 \gamma_{n}^{2}}\right\}
\end{aligned}
$$$$
\alpha \exp \left\{\frac{-1}{2}\left[\frac{\sum_{i=1}^{x}\left[w_{m}\left(t_{i-1}\right)\right]^{2} \delta t}{d_{m}^{2}}+\frac{1}{\gamma_{m}^{2}}\right] \lambda_{m}^{2}\right\}
$$$$
\left.\times \exp \left\{\left[\frac{\sum_{i=1}^{x} \delta A_{m}\left(t_{i}\right) w_{m}\left(t_{i-1}\right)}{d_{m}^{2}}+\frac{\beta_{m}}{\gamma_{m}^{2}}\right] \lambda_{m}^{2}\right\}\right\}
$$$$
\times\left\{\exp \left\{\frac{-1}{2}\left[\frac{\sum_{i=1}^{x}\left[w_{n}\left(t_{i-1}\right)\right]^{2} \delta t}{d_{n}^{2}}+\frac{1}{\gamma_{n}^{2}}\right] \lambda_{n}^{2}\right\}\right.
$$$$
\left.\times \exp \left\{\left[\frac{\sum_{i=1}^{x} \delta A_{n}\left(t_{i}\right) w_{n}\left(t_{i-1}\right)}{d_{n}^{2}}+\frac{\beta_{n}}{\gamma_{n}^{2}}\right] \lambda_{n}^{2}\right\}\right\}
$$$$
\left.\alpha \exp \left\{-\frac{\left[\lambda_{m}-\frac{\gamma_{m}^{2} \sum_{i=1}^{x} \delta A_{m}\left(t_{i}\right) w_{m}\left(t_{i-1}\right)+\beta_{m} d_{m}^{2}}{\gamma_{m}^{2} \sum_{i=1}^{x}\left[w_{m}\left(t_{i-1}\right)\right]^{2} \delta t+d_{m}^{2}}\right]^{2}}{\frac{2 d_{m}^{2} \gamma_{m}^{2}}{\gamma_{m}^{2} \sum_{i=1}^{x}\left[w_{m}\left(t_{i-1}\right)\right]^{2} \delta t+d_{m}^{2}}}\right\}\right\}
$$$$
\times\left\{\exp \left\{-\frac{\left[\lambda_{n}-\frac{\gamma_{n}^{2} \sum_{i=1}^{x} \delta A_{n}\left(t_{i}\right) w_{n}\left(t_{i-1}\right)+\beta_{n} d_{n}^{2}}{\gamma_{n}^{2} \sum_{i=1}^{x}\left[w_{n}\left(t_{i-1}\right)\right]^{2} \delta t+d_{n}^{2}}\right]^{2}}{\frac{2 d_{n}^{2} \gamma_{n}^{2}}{\gamma_{n}^{2} \sum_{i=1}^{x}\left[w_{n}\left(t_{i-1}\right)\right]^{2} \delta t+d_{n}^{2}}}\right\}\right\}
$$ 
for $\mathrm{t}_{x}$

$$
\begin{aligned}
& \alpha N\left(\beta_{m, n}\left(t_{x}\right), \gamma_{m, n}^{2}\left(t_{x}\right)\right) \\
& \beta_{m}\left(t_{x}\right)=\frac{\left(\gamma_{m}^{2} \sum_{i=1}^{x} \delta A_{m}\left(t_{i}\right) w_{m}\left(t_{i-1}\right)+\beta_{m} d_{m}^{2}\right)}{\left(\gamma_{m}^{2} \sum_{i=1}^{x}\left[w_{m}\left(t_{i-1}\right)\right]^{2} \delta t+d_{m}^{2}\right)} \\
& \beta_{n}\left(t_{x}\right)=\frac{\left(\gamma_{n}^{2} \sum_{i=1}^{x} \delta A_{n}\left(t_{i}\right) w_{n}\left(t_{i-1}\right)+\beta_{n} d_{n}^{2}\right)}{\left(\gamma_{n}^{2} \sum_{i=1}^{x}\left[w_{n}\left(t_{i-1}\right)\right]^{2} \delta t+d_{n}^{2}\right)} \\
& \gamma_{m}^{2}\left(t_{x}\right)=\frac{d_{m}^{2} \gamma_{m}^{2}}{\gamma_{m}^{2} \sum_{i=1}^{x}\left[w_{m}\left(t_{i-1}\right)\right]^{2} \delta t+d_{m}^{2}} \\
& \gamma_{n}^{2}\left(t_{x}\right)=\frac{d_{n}^{2} \gamma_{n}^{2}}{\gamma_{n}^{2} \sum_{i=1}^{x}\left[w_{n}\left(t_{i-1}\right)\right]^{2} \delta t+d_{n}^{2}}
\end{aligned}
$$

Here, $\lambda_{m, n} \mid \delta A_{m, n}\left(t_{x}\right), w_{m, n}\left(t_{x-1}\right), \beta_{m, n}\left(t_{x}\right), \gamma_{m, n}^{2}\left(t_{x}\right)$ are as expressed in the proposition. Note that the last equation is characterized by the normal density function. Thus $\left(\lambda_{m, n} \mid \delta A_{m, n}\left(t_{x}\right), w_{m, n}\left(t_{x-1}\right)\right)$ has a normal posterior distribution with $\left(\beta_{m, n}\left(t_{x}\right), \gamma_{m, n}^{2}\left(t_{x}\right)\right)$ as mean and variance, respectively. To be specific, as it is a hybrid configuration, two sets of means and variances will form due to different sets of machine $(m, n)$, which have been clearly justified in the above proof.

\section{Numerical case study}

To evaluate the proposed methodology implemented for two scenarios, we evaluate the precision of its predictions using input data proposed by Hao et al [30]. In addition, we evaluate the absolute quality of our methodology by implementing it on a real world time data for parallel machines as illustrated in Chalklevitch and Glass [36].

To manufacture various parts and to acquire the parameters related to degradation, the following section explains the experimental set-up for conducting the tests. We first present a hypothetical stamping system with five identical stamping machines working in parallel configuration and later we deliberate the parameters of a real world scenario. In this area of analysis, degradation signals, degradation coefficient, instantaneous degradation rate and a few other parameters associated with degradation are taken into account, as mentioned in Chen and Jin [37]. Parameters utilized to initiate the degradation signals of a unit of workload, are considered altogether for expressing the real world characteristics, as the quantity of parts taken per unit time. Considering all the machines, each of them has its mean of "degradation coefficient" established in advance, i.e., $\beta_{1, \ldots,} \beta_{2}$, which equals $5.97 \times 10^{-8}$ inch per part.
Likewise, considering each machine, its diffusion parameter of the Brownian motion error, i.e., $d_{1}, \ldots, d_{5}$, is found to be $2.03 \times 10^{-5}$ inch per unit time and is marked. For representation of the actual manufacturing settings, for each machine, the failure threshold is recognized as 0.004 . This numerical case study states about the assignment of each machine with a peak workload of 1500 parts per day with the demand remaining fixed at 6000 parts per day. In this case, a single day has been considered as the measure of the decision epoch, i.e., the unit time. If the ongoing operation of a machine is interrupted by its malfunctioning, it has to be repaired for which a fixed amount of time has been considered which equals to a minimum of one decision epoch. Requirement of an excess repair time, regarding any multiple of a day, may arise. Nevertheless, if the number of machines that fail simultaneously exceeds one, the demand would no longer be in a stage to be satisfied. As mentioned earlier, the real time scenario illustrated in Chalklevitch and Glass [36], having the same degradation parameters has been tested. Two sets of machines, namely A and B, are considered where each set consists of 6 machines and 4 machines, respectively. These machines are in parallel configuration amongst themselves and in their respective sets, which are further in hybrid configuration with each other. The demand that has been set in order to be completed on a daily basis, is around 5000 parts per day.

\section{Experimentation}

In this section, the implementation of the suggested dynamic workload adjustment methodology, with the pair of other scenarios, is enumerated. The computation was carried out on a $P C$ with Intel Core i5-4302Y $(1.6 \mathrm{GHz}, 512$ KB L2 cache) having Windows 10 Home operating system with 4 GB RAM. In SC-1, i.e., Scenario-1, all operational units were assigned with equal workloads. However, in SC2 , the workloads were assigned randomly as stated in Hao et al [30]. Combinations of workload assignments demonstrated by the functional units under SC-2 were unique, out of which random workload selection has been considered for allotting the workloads on the particular units. For the performance analysis, we considered two key performance indices (KPIs): (1) the percentage of time in which a minimum of a single machine has faced failure and is being repaired and (2) the percentage decrement in production. It is also noted that in the following set-up, the demand has to be left unsatisfied, in case the number of machines under repair exceeds unity, which may ultimately lead to production loss. To analyze the scenarios under a couple of factors, the performance of machines under repair time (1, 3 and 5 days) was considered as the initial factor. The second factor was considered to be the coefficient of variation $(\mathrm{CV})$ of the prior distribution of the degradation coefficient $\lambda_{m}$, where $\mathrm{CV}$ denotes the ratio of standard deviation, i.e., $\gamma_{m}$ to the mean i.e. $\beta_{m}$. Thus, for each and 

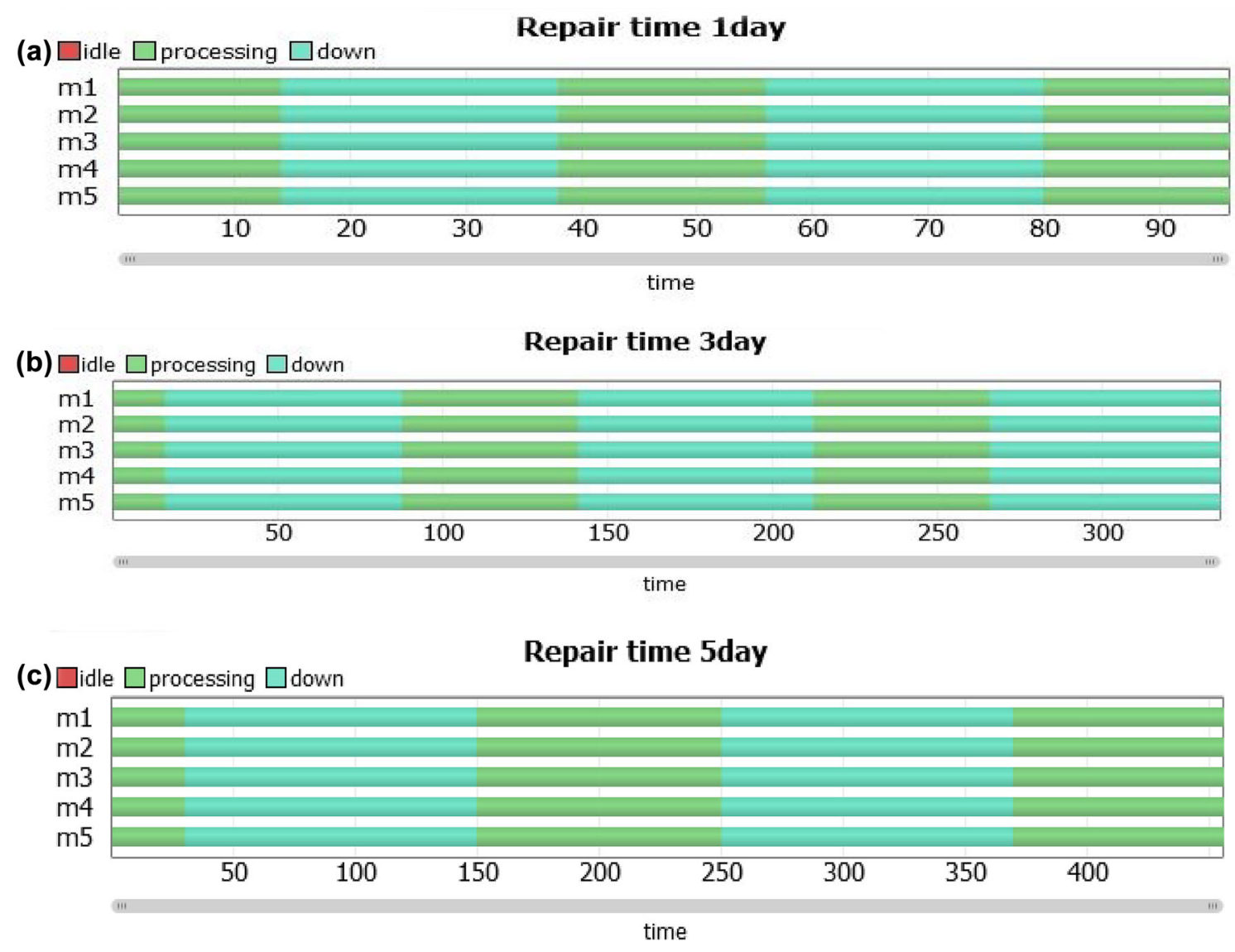

Figure 5. (a-c) Scenario-1 (equally distributed arrangement of workload).

every $m$ unit, its CV of $\lambda_{m}$ is defined as $\left(\gamma_{m} / m\right)$. Hence, the variation of the degradation coefficient among various units is brought about by CV. By this, it is observed that there is a reduction in the unit-to-unit variability with the decrement of CV. In this context, all machines have equal $\beta_{m}=$ $5.97 \times 10^{-8}$ inch per part. Hence, if $\mathrm{CV}$ is small, then the actual values of $\lambda$ of various machines are closer to one another, and that is why they are observed to fail at the same time on assigning similar workloads to them. The observation was carried out under four separate levels of CVs: $(1 / 3),(1 / 6),(1 / 9)$ and (1/12). For a total of $3 \times 4=12$ conditions, 30 experiments were performed. Similarly, to evaluate the real world scenario, the ratio $\mathrm{R}$ was calculated using four different instances $(\mathrm{R}=0.5, \mathrm{R}=1, \mathrm{R}=1.5$ and $\mathrm{R}$ $=2$ ) on two different sets of machines for the above mentioned two key performance indices, time intervals and different levels of $\mathrm{CV}$. Here, $\mathrm{R}$ is defined as the ratio of the total workload of the secondary set of machines and the average workload of the primary parallel machines.

Table 1 depicts the comparison between the parallel configuration used in this paper and the same that is present in Hao et al [30]. By comparing percentage time when more than one machine is under repair and loss of production in both the configurations, one can observe that our proposed methodology along with SC-1 and SC-2 is better than the one present in Hao et al [30].

Table 2 compares parallel and hybrid configurations. The parameters such as percentage time when more than one machine is under repair, loss of production on three different scenarios (SC-1, SC-2 and PM) have been used. The comparison and verification of these scenarios for parallel and hybrid configurations were observed under four different levels of CVs i.e., (1/3), (1/6), (1/9) and (1/12) each for repair time of 1,3 and 5 days. In this paper, our main aim is to minimize the throughput time so as to achieve the required dynamic workload adjustment strategy for fulfilling the production requirement. In table 1 , when we compare parallel and hybrid configurations, by verifying percentage time when more than one machine is under repair and loss of production on the given scenarios, i.e., SC-1, SC-2 and PM, we conclude that for the repair time of 3 and 5 days, hybrid configuration is more effective and efficient because of minimum percentage time when more than one machine is under repair and loss of production. But the scenario is slightly different for the repair time of 1 day as the efficiency in parallel configuration is comparable with that of hybrid configuration and is also better in some levels of CVs. This is because of the frequency of the 

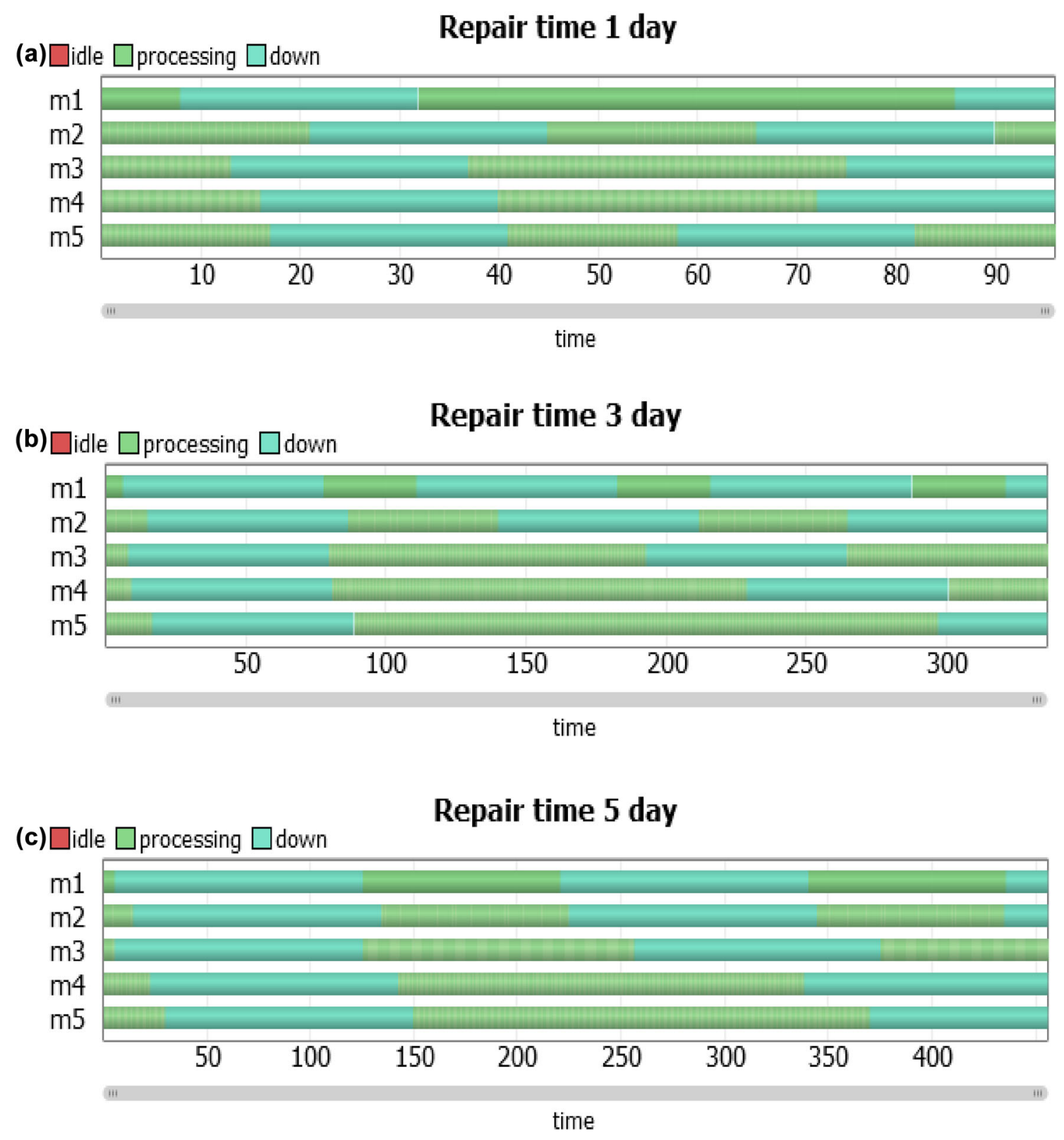

Figure 6. (a-c) Scenario-2 (random arrangements of workload).

machine failure, i.e., for 1 day, the frequency that a machine would fail is less and so the variation in the values is less, whereas in case of 3 and 5 days, the frequency of machine failure is more because of more number of days. Hence, the variation in the values is significantly larger, so both the configurations in case of 3 and 5 days can be easily distinguished to find which one is better.

Tables 3 and 4 articulates the results for real case scenario. After analyzing the results i.e., the percentage time when more than one machine is under repair and loss of production for all four different ratios of $\mathrm{R}(\mathrm{R}=0.5, \mathrm{R}=1$, $R=1.5$ and $R=2$ ), it is clearly noticeable that more number of breakdown occurs when the value of $\mathrm{R}$ is less. From the results of tables 2 and 3, conclusion can be made that a similar kind of pattern which was seen in the above tables i.e., tables 1 and 2 is observed. Also, by observing the trends for the percentage time when more than one machine is under repair, it is determined that, when the system follows the ratio of 0.5 , more machines fall under repair at the same time in comparison to when the system follows ratio equals 2 . This is because, when the ratio is less the amount of workload given to the secondary set of machines is less. Therefore the load on primary machines is more which results in more failure of machines (table 5).

The identified resources from propositions and their effect on the performance manufacturing systems on the 

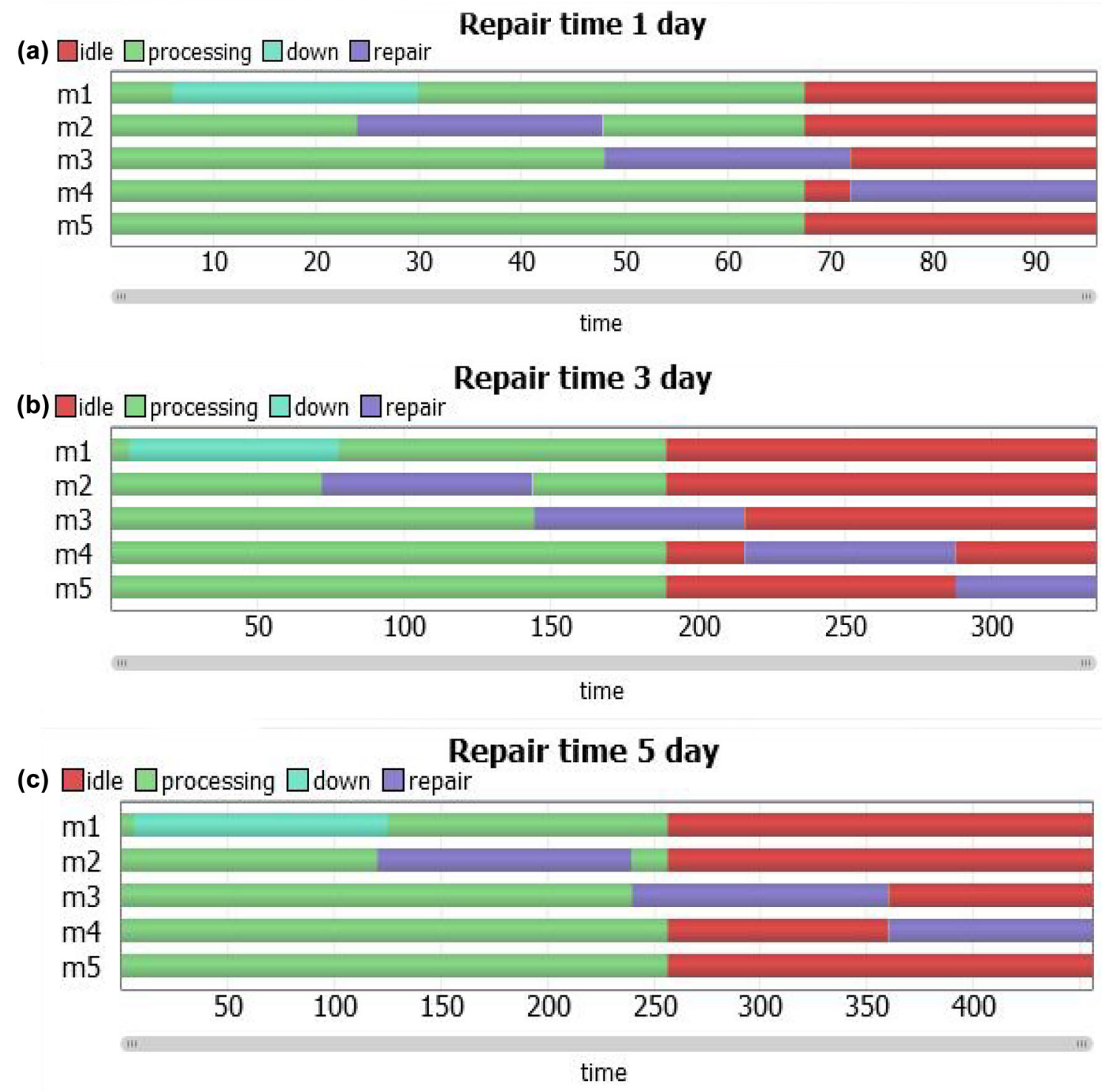

Figure 7. (a-c) Proposed methodology (systematic arrangement of workload).

basis of performance measures are considered in the form of boxplots. To be part of it, a simulated environment has been created with the help of FlexSim simulation tool. The simulation analysis was carried out and the results of the considered case, i.e., the average maximum completion of the workloads (make span) have been illustrated in figures 5, 6, 7, 8, 9 and 10 as gantt charts.

The above figures $5(\mathrm{a}-\mathrm{c})$ show the simulation program outcomes, i.e., for SC-1, for repair time 1, 3 and 5 days. The outcomes of figures $5(\mathrm{a}-\mathrm{c})$ clearly conclude that in the cases of all the three repair times, the workload allotted to the machines was not completed as a whole, which led to the production loss. From figures $5(\mathrm{a}-\mathrm{c})$, it is observed that, as equal workloads were allotted to the machines, they ultimately failed altogether at the same time-period.
Figures $6(\mathrm{a}-\mathrm{c})$ show the performance of simulation program of SC-2, for repair time 1, 3 and 5 days. From the results attained, it is clear that similar to the case of SC-1, here also, in all the three figures, the workload which was assigned, was not completed as a whole, for which loss of production was the outcome. However, when a comparative study was made between figures 6 and 10, one could clearly comprehend that the loss of production was found less in SC-2 which clearly defines that when the random workload was assigned, the tendency of unit failure overlap was restricted to a certain level.

Figures $7(a-c)$ represent the results of the simulation program with proposed methodology, for repair time 1, 3 and 5 days. From the results obtained, a difference was observed in comparison with figures 6 and 7, i.e., a change 


\section{(a)}

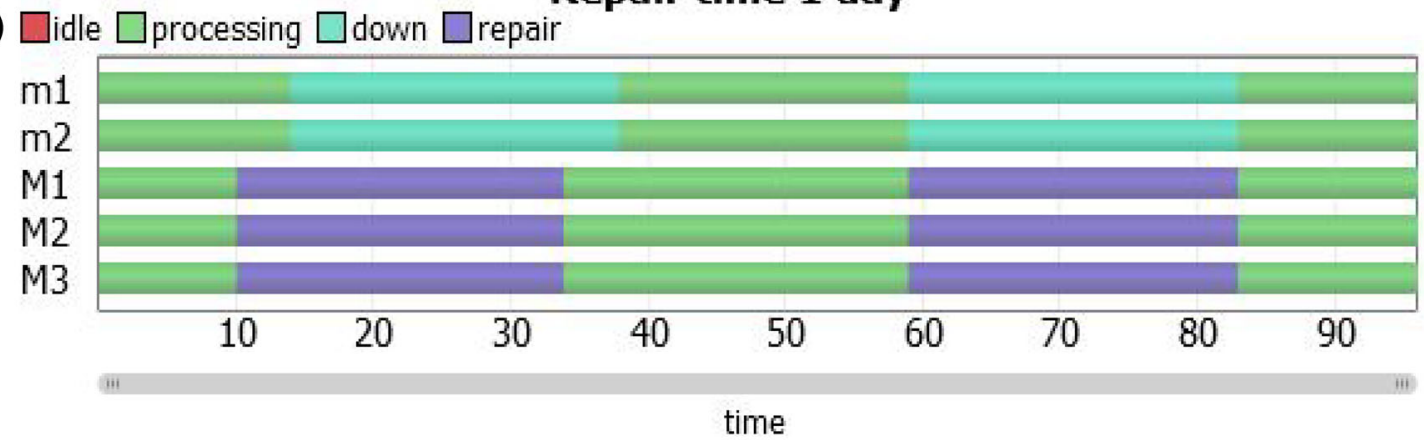

\section{Repair time 1 day}
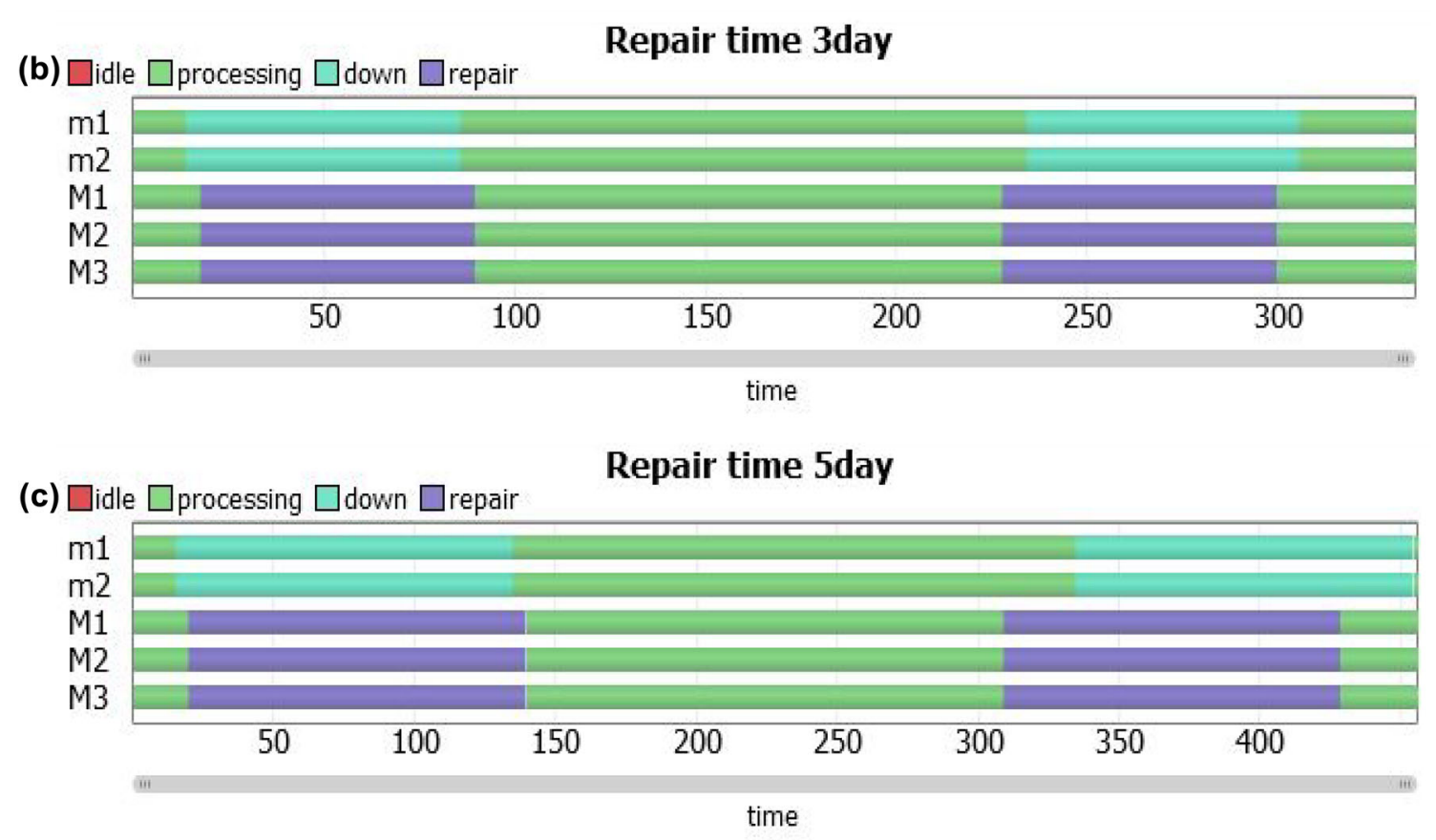

Figure 8. (a-c) Scenario-1 (equally distributed arrangement of workload).

in the color of the graphs which shows the idle period of the machines. As in the proposed methodology, a dynamic workload adjustment was carried out, the tendency of unitto-unit failure overlap was purely cured which resulted in the completion of the assigned workload.

As an extension of our work, we ran the simulation for hybrid configuration of the system. The results which were obtained from the simulation were quite similar to parallel configuration, but the tendency of machine failure was less.

The above figures $8(\mathrm{a}-\mathrm{c})$ depict results of the simulation program, i.e., for SC-1, for repair time 1, 3 and 5 days. From the graph, it is observed that as the simulation was following SC-1, all the machines tend to fail at a same period. However, due to the hybrid configuration, the overlap of machine failure was in a pair of two and three. Due to which the make span of SC-1 of the hybrid was better than parallel configuration.

Figures $9(\mathrm{a}-\mathrm{c})$ show the performance of simulation program of SC-2, for repair time 1, 3 and 5 days. From figures 9(b) and (c) one can see that the machine becomes idle which indicates that the amount of workload, which was assigned to a parallel pair of two machines (i.e., M1 and M2), is completed, due to which the overall loss of production was found to be less in comparison to figure 8(a) and (b) and figure 9(a).

Figures $10(a-c)$ demonstrate the results of the simulation program with the proposed methodology, for repair time 1, 3 and 5 days. Here also, similar to the proposed methodology of parallel configuration, the assigned workload was completed but the time for the completion was less than that of the parallel configuration. To be more specific as the machines were in a pair of 2 and 3, the amount of workload was divided into two sets according to the maximum capacity of the pairs of machines, which is reflected clearly in figures $10(\mathrm{a}-\mathrm{c})$.

In this subsection, we compare the performance of figures 5, 6 and 7 with figures 8,9 and 10. The summary of 

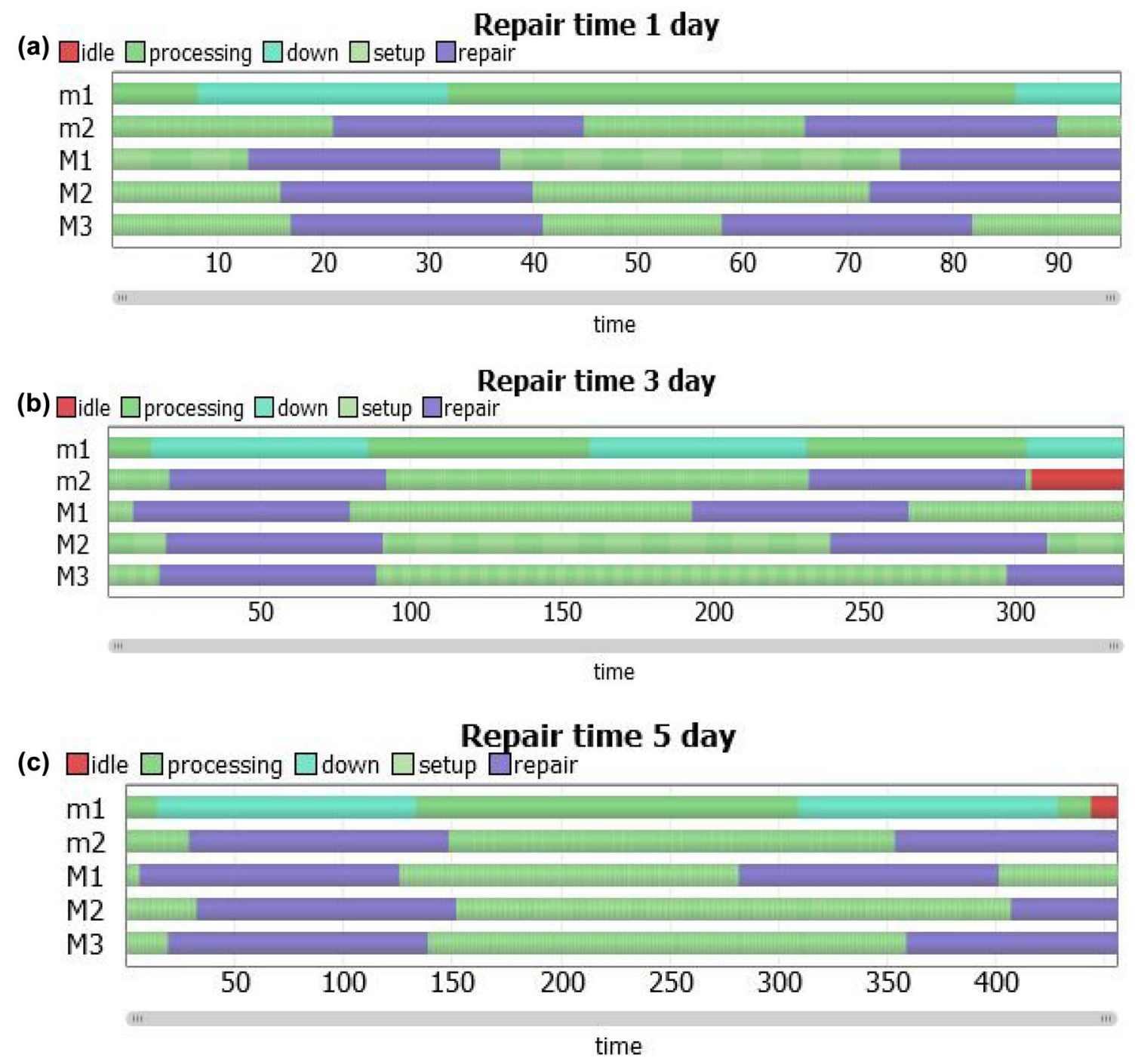

Figure 9. (a-c) Scenario-2 (random arrangements of workload).

the comparison was that one could see how hybrid configuration is a better option than parallel configuration. In the hybrid configuration, idle time was found for each of the scenarios which was not seen in the parallel configuration. From figures 7 and 10, a conclusion can be made that in both the cases a certain amount of time is found to be idle which proves that our proposed methodology is more beneficial than other scenarios.

To present the health status of machines, graphs have been plotted between the degrading coefficient of the machines with respect to the number of products being manufactured. Here, we have considered the region in which the machines start degrading and fail for the first time. The degrading coefficient of the machines are presented in the $y$-axis ranging between 0 and 1 i.e., 1 representing the best condition whereas 0 for failure of machines. The $\mathrm{x}$-axis presents the number of products that have been manufactured corresponding to the health status.
The graphs here signify that the health status of the machines is inversely proportional to the number of products.

As shown in figure 11, in case of $\mathrm{SC}-1$ for parallel configuration, all the five machines fail at the same time because of the equal distribution of the workload. When an appropriate observation of the graph is carried out, it has been observed that between the product range of 450 and 620 , the degradation is occurring at a very slow pace and it is also visible that the line is not a straight but curved at a certain point from which we can conclude that the degradation of the machines occurs at a variable pace instead of a constant rate. A single line has been plotted for all the machines as they have a similar type of health status due to equal distribution of workload. For figure 12, representing SC-2 configuration, all the five machines fail at different time intervals because of the random distribution of the workload. On 


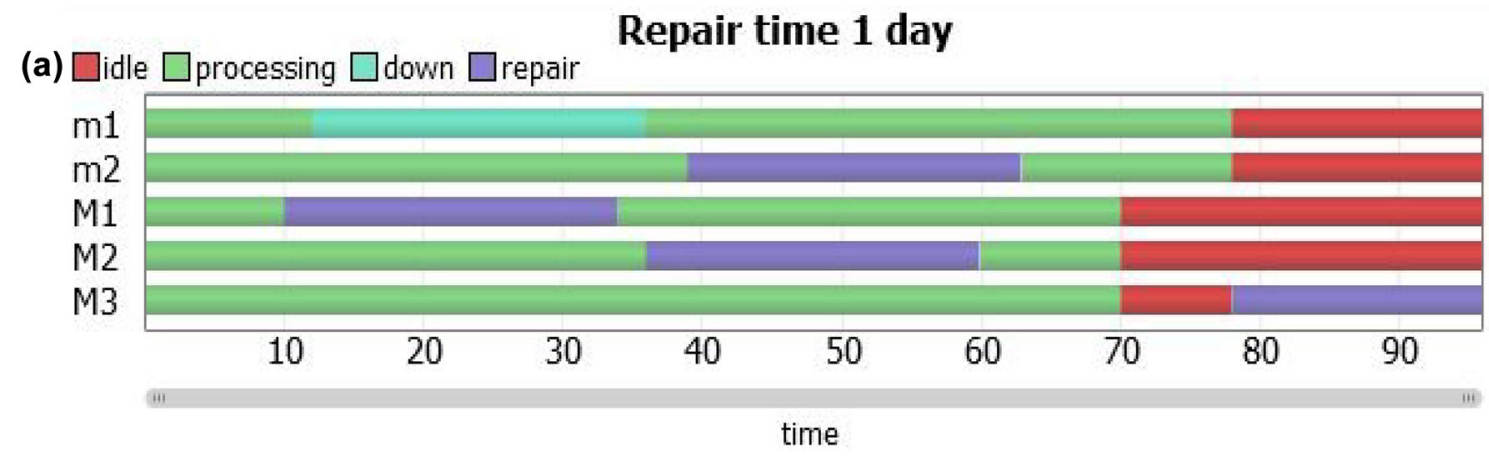

(b) $\square$ idle $\square$ processing $\square$ down $\square$ repair

\section{Repair time 3 day}

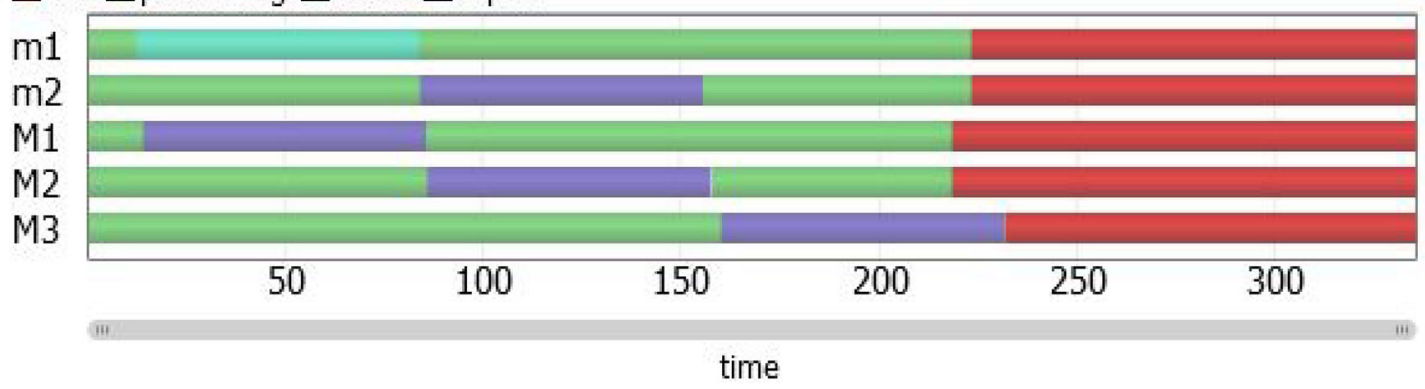

(c) $\square$ idle $\square$ processing $\square$ down $\square$ repair

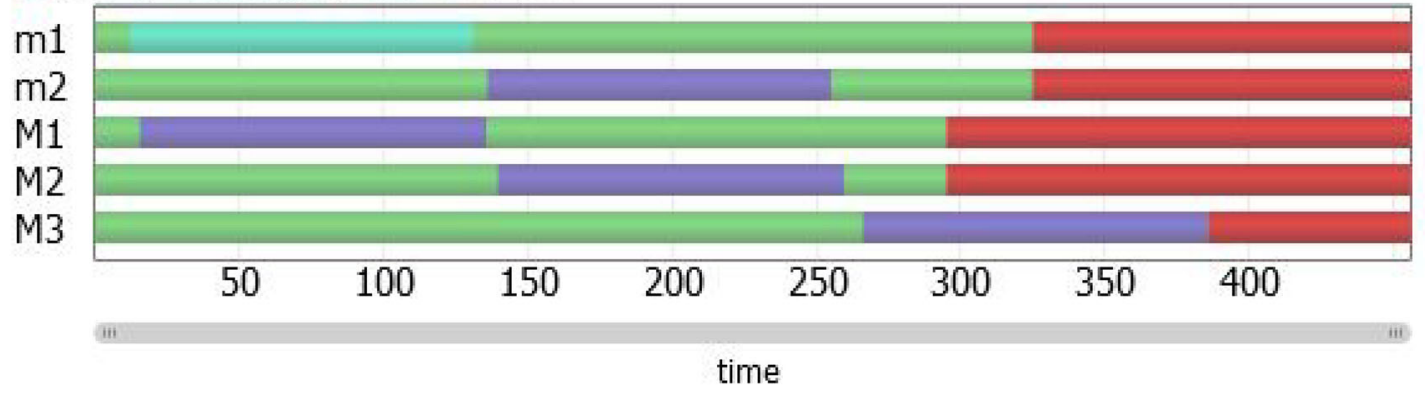

Figure 10. (a-c) The proposed methodology (systematic arrangement of workload).

appropriate observation of the graph, it is visible that between the product range of 250 and 1000, a measurable change in the degradation rate has occurred. Figure 13 describes the health status of proposed model for parallel configuration i.e., PM. From the results of figure 13, a clear conclusion can be made that the demand which was set as 6000 products is reached before all the machines got failed. By analyzing the graph, one can see that machine no. 3, 4, 5 i.e., M3, M4, M5 are having a health status coefficient of $0.08421,0.1855$, 0.1984 , respectively which state that much more work can be carried out on it. Another conclusion that is observed over here is that there is no intersection of the lines which states that no machines are failing in group thus improving the production rate.

Figure 14, represents the health status for SC-1 in hybrid configuration. Here, for the hybrid five machines are working in a combination of two sets such that each set consists of two and three machines, respectively. It is observed that the machines which are working in their respective sets will always fail at the same time due to equal distribution of the workload, whereas the failure time of both the sets can be different. When an appropriate observation of the graph is carried out, it has been observed that the degradation rate for the products ranging from 220 to 800 is different as the degradation is occurring at a variable pace and is not uniform, thus creating a sudden curve structure in the graph. As shown in figure 15, representing SC-2 it can be observed that the degradation rate of a machine is varying and all the five machines fail at different time intervals because of the random distribution of the workload. Upon an appropriate observation of the graph, one can see that between the product range of 600 and 2000, a measurable change in the degradation rate has 


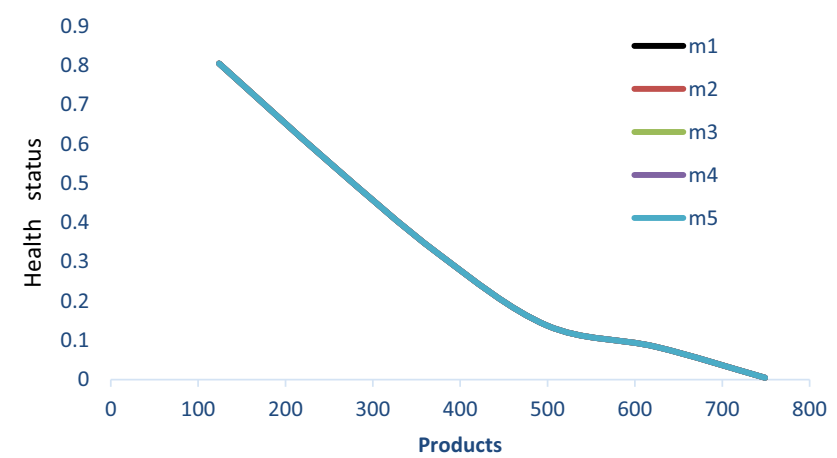

Figure 11. Scenario-1 (parallel configuration - health status vs products).

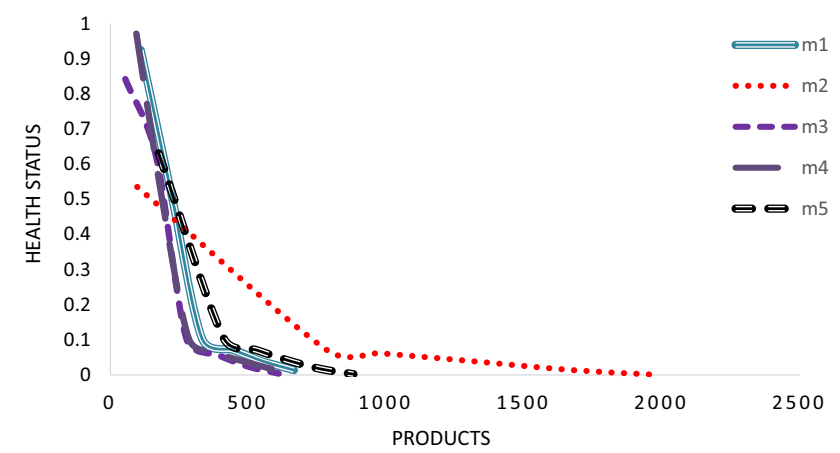

Figure 12. Scenario-2 (parallel configuration - health status vs products).

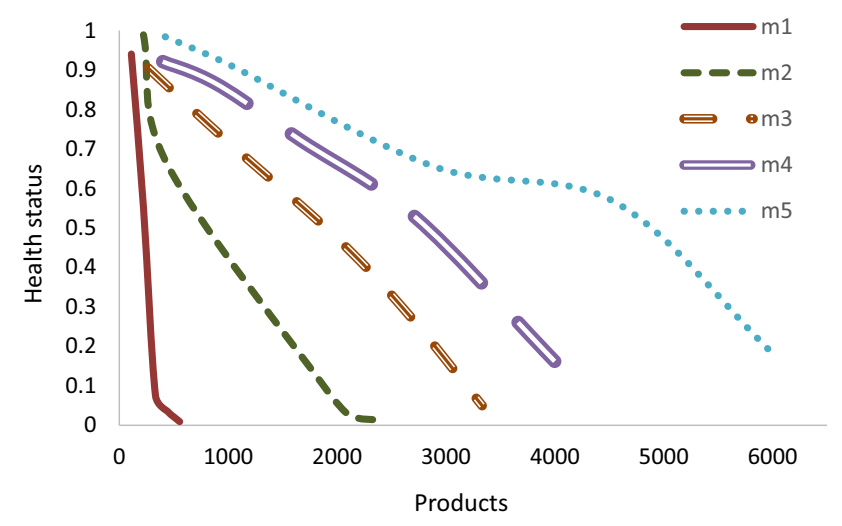

Figure 13. The proposed methodology (parallel configuration health status vs products).

occurred. Figure 16 represents the health status of our proposed model for hybrid configuration i.e., Pm. From the results of figure 16, a clear conclusion can be made that the demand which was set as 6000 products is reached before all the machines failed. By observing the graph, one can see that machine no. 1, 2, 5 i.e., M1, M2, M5 are having a health status coefficient of $0.0993,0.0182,0.1185$, respectively which signifies that much more work can be carried out on it. Another observation can be drawn from

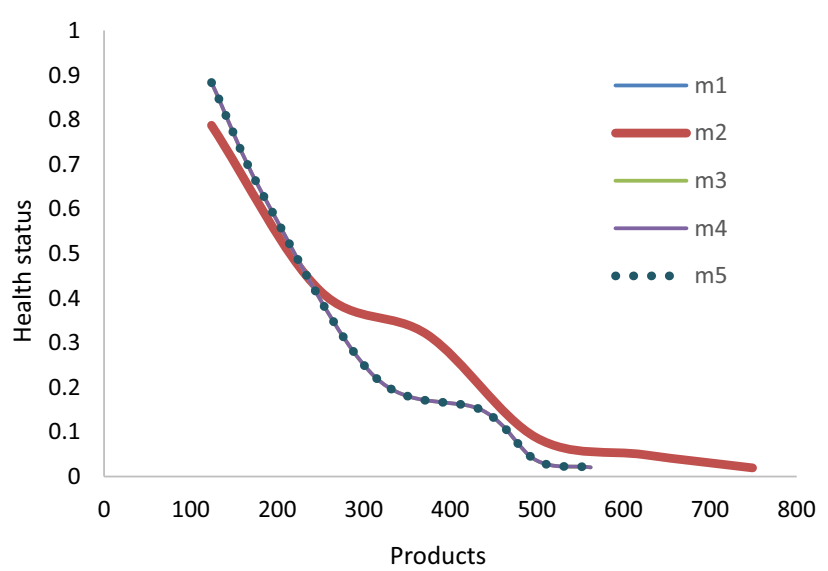

Figure 14. Scenario-1 (hybrid configuration - health status vs products).

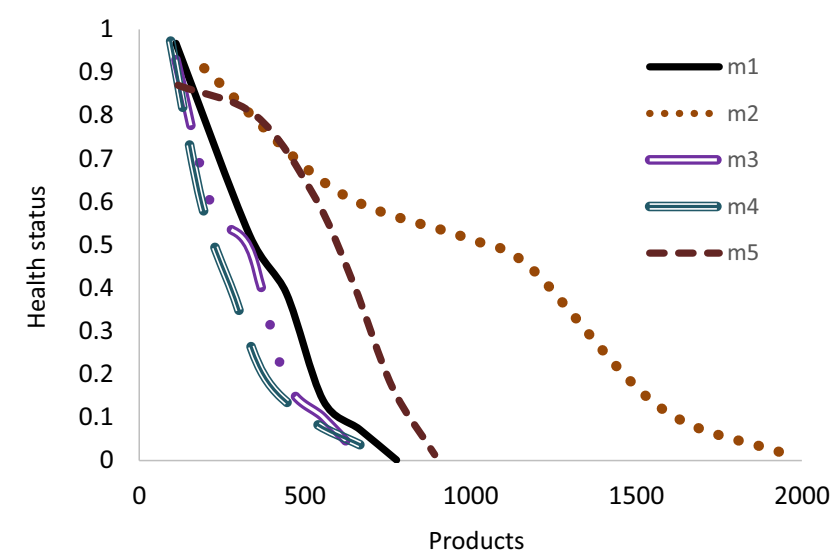

Figure 15. Scenario-2 (hybrid configuration - health status vs products).

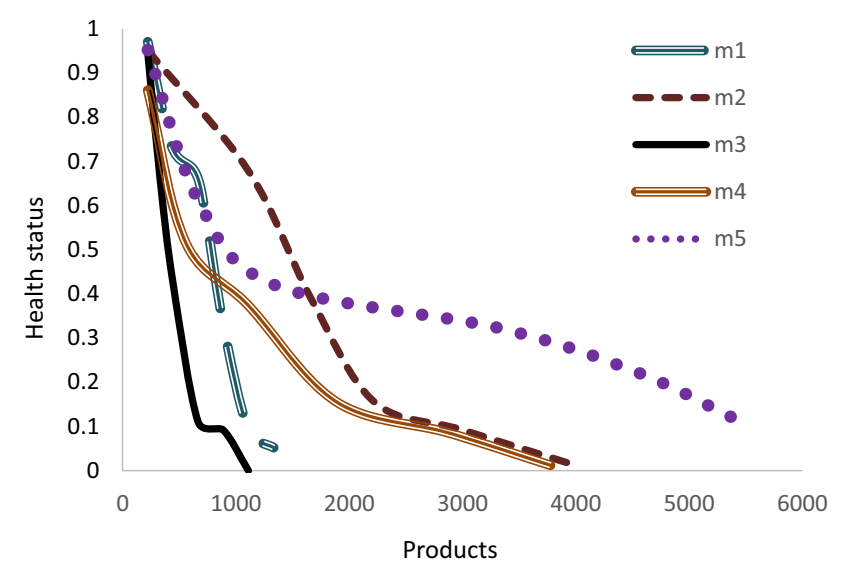

Figure 16. The proposed methodology (hybrid configuration health status vs products).

the above graph regarding the efficiency of parallel and hybrid configuration in case of our proposed methodology which concluded that the efficiency of hybrid configuration is much higher than the parallel configuration. 


\section{Conclusion and future work}

In this paper, regarding real life adjustment of industrial units, we concentrate on dynamic workload adjustment strategy for controlling the degradation process of each unit. A Bayes scheme is taken into account, which deals with real time degradation monitoring information, for carrying out the upgradation of the preliminary distribution of the degradation coefficients. After this, the dynamic workload assignment policy is obtained by our method at each decision epoch and targets at averting the cases of unit failure overlap. A numerical study is organized and a contrast between the pair of scenarios, the first one with evenly distributed workloads and the second one with randomly distributed workloads, is brought about for demonstrating our method. It is clear from the outcomes that our method is certainly better than these two scenarios, under different situations, regarding the prevention of the overlap of unit failures and satisfaction of the production demand. Moreover, the merit of our proposed methodology shows its peak with the increment of repair time or with the decrement of unit-to-unit variability.

The main contribution of our paper is to open a different approach of research that concentrates on the dynamic adjustment of the workloads allocated to individual units to control the failure time and to check the degradation rate for obtaining a satisfactory performance of the system. The topic that we have chosen had previously been used in case of parallel configuration, and we have further extended it by considering the serial-parallel or commonly termed as the hybrid configuration. The literature about our context indicates the self-progressing degradation process, which in turn, is important for the prediction of residual life. On the contrary, the problems on which we have worked, highlights the relation between degradation rate of a unit and workload assigned to a unit i.e., both are directly proportional. Thus, by adjusting the workload, the degradation rate can be knowingly controlled and also the residual life can be predicted to cater the engineering demand.

For the future work, we will add on the concept different part type jobs instead of identical ones and will find the residual life of each machine for the betterment of the industrial sectors.

\section{References}

[1] Kaiser K A and Gebraeel N Z 2009 Predictive maintenance management using sensor-based degradation models. IEEE Trans. Systems Man Cybern. A Syst. Hum. 39: 840-849

[2] Wakui T, Yamaguchi K, Hashizume T, Outa E and Tanzawa Y 1999 Effect of operating methods of wind turbine generator system on net power extraction under wind velocity fluctuations in fields. Renew. Energy 16: 843-846
[3] Suzuki K, Yamada T, Ishii M, Shibata T and Mino S 2007 High-speed optical $1 \times 4$ switch based on generalized machzehnder interferometer with hybrid configuration of silicabased PLC and lithium niobate phase-shifter array. IEEE Photonics Technol. Lett. 19: 674-676

[4] Otoo M and Collins J 2011 Industrial Production and Capacity Utilization: The 2010 Annual Revision.

[5] Lu C J and Meeker W O 1993 Using degradation measures to estimate a time-to-failure distribution. Technometrics 35: 161-174

[6] Gebraeel N Z, Lawley M A, Li R and Ryan J K 2005 Residual-life distributions from component degradation signals: A Bayesian approach. IIE Trans. 37: 543-557

[7] Martin K F 1994 A review by discussion of condition monitoring and fault diagnosis in machine tools. Int. J. Mach. Tools Manuf. 34: 527-551

[8] Fararooy S and Allan J 1995 On-line condition monitoring of railway equipment using neural networks. In: IEE Colloquium on 'Advanced Condition Monitoring Systems for Railways'. p 2

[9] Dimla D E 1999 Artificial neural networks approach to tool condition monitoring in a metal turning operation. In: 7 th IEEE International Conference on Emerging Technologies and Factory Automation, Proceedings, ETFA'99, IEEE. 1: 313-320

[10] Thorsen O V and Dalva M 1999 Failure identification and analysis for high-voltage induction motors in the petrochemical industry. IEEE Trans. Ind. Appl. 35: 810-818

[11] Wang W B 2000 A model to determine the optimal critical level and the monitoring intervals in condition-based maintenance. Int. J. Prod. Res. 38: 1425-1436

[12] Huang J, Zuo M J and Wu Y 2000 Generalized multi-state k-out-of-n: G systems. IEEE Trans. Reliab. 49: 105-111

[13] Zuo M J and Tian Z 2006 Performance evaluation of generalized multi-state k-out-of-n systems. IEEE Trans. Reliab. 55: 319-327

[14] Chao M T, Fu J C and Koutras M V 1995 Survey of reliability studies of consecutive-k-out-of-n: $\mathrm{F}$ and related systems. IEEE Trans. Reliab. 44: 120-127

[15] Bian L and Gebraeel N 2014 Stochastic modeling and realtime prognostics for multi-component systems with degradation rate interactions. IIE Trans. 46: 470-482

[16] Hao L, Gebraeel N and Shi J 2015 Simultaneous signal separation and prognostics of multi-component systems: The case of identical components. IIE Trans. 47: 487-504

[17] Li J 2004 Modeling and analysis of manufacturing systems with parallel lines. IEEE Trans. Autom. Control 49: 1824-1832

[18] Li J 2005 Overlapping decomposition: A system-theoretic method for modeling and analysis of complex manufacturing systems. IEEE Trans. Autom. Sci. Eng. 2: 40-53

[19] Sloan T W and Shanthikumar J G 2000 Combined production and maintenance scheduling for a multiple-product, single-machine production system. Prod. Oper. Manag. 9: 379-399

[20] Sloan T W and Shanthikumar J G 2002 Using in-line equipment condition and yield information for maintenance scheduling and dispatching in semiconductor wafer fabs. IIE Trans. 34: 191-209

[21] Zhou J, Djurdjanovic D, Ivy J and Ni J 2007 Integrated reconfiguration and age-based preventive maintenance decision making. IIE Trans. 39: 1085-1102 
[22] Lu B, Zhou X and Li Y 2016 Joint modeling of preventive maintenance and quality improvement for deteriorating single-machine manufacturing systems. Comput. Ind. Eng. 91: 188-196

[23] Deep K and Singh P K 2016 Dynamic cellular manufacturing system design considering alternative routing and part operation tradeoff using simulated annealing based genetic algorithm. Sādhanā 41: 1063-1079

[24] Mosallam A, Medjaher K and Zerhouni N 2016 Data-driven prognostic method based on Bayesian approaches for direct remaining useful life prediction. J. Intell. Manuf. 27: 1037-1048

[25] Peng W, Li Y F, Yang Y J, Mi J and Huang H Z 2017 Bayesian degradation analysis with inverse Gaussian process models under time-varying degradation rates. IEEE Trans. Reliab. 66: 84-96

[26] Alaswad S and Xiang Y 2017 A review on condition-based maintenance optimization models for stochastically deteriorating system. Reliab. Eng. System Saf. 157: 54-63

[27] Arasteh A 2016 Stochastic decision making in manufacturing environments. J. Ind. Syst. Eng. 9: 17-34

[28] Asghar E, uz Zaman U K, Baqai A A and Homri L 2018 Optimum machine capabilities for reconfigurable manufacturing systems. Int. J. Adv. Manuf. Technol. 95: 4397-4417

[29] Rodriguez M A and Buyya R 2018 Scheduling dynamic workloads in multi-tenant scientific workflow as a service platforms. Fut. Gen. Comput. Syst. 79: 739-750
[30] Hao L, Liu K, Gebraeel N and Shi J 2015 Controlling the residual life distribution of parallel unit systems through workload adjustment. IEEE Trans. Autom. Sci. Eng. 14: 1042-1052

[31] Chandraa A, Ahsana M, Lahiria S, Panigrahia S, Manupati V K and Costab E 2017 Degradation Modeling to Predict the Residual Life Distribution of Parallel Unit Systems on Benchmark Instances. In: Proceedings of the World Congress on Engineering (Vol. 2)

[32] Elwany A and Gebraeel N 2009 Real-time estimation of mean remaining life using sensor-based degradation models. J. Manuf. Sci. Eng. 131: 051005

[33] Gebraeel N Elwany A and Pan J 2009 Residual life predictions in the absence of prior degradation knowledge. IEEE Trans. Reliab. 58: 106-117

[34] Liu K, Gebraeel N Z and Shi J 2013 A data-level fusion model for developing composite health indices for degradation modeling and prognostic analysis. IEEE Trans. Autom. Sci. Eng. 10: 652-664

[35] Bian L and Gebraeel N 2012 Computing and updating the first-passage time distribution for randomly evolving degradation signals. IIE Trans. 44: 974-987

[36] Chakhlevitch K and Glass CA 2009 Scheduling reentrant jobs on parallel machines with a remote server. Comput. Oper. Res. 36: 2580-2589

[37] Chen Y and Jin J 2006 Quality-oriented-maintenance for multiple interactive tooling components in discrete manufacturing processes. IEEE Trans. Reliab. 55: 123-134 